\title{
Relative effect of nodes and filaments of the cosmic web on the quenching of galaxies and the orientation of their spin
}

\author{
Nicola Malavasi®, Mathieu Langer®, Nabila Aghanim, Daniela Galárraga-Espinosa®, and Céline Gouin® \\ Université Paris-Saclay, CNRS, Institut d'Astrophysique Spatiale, 91405 Orsay, France \\ e-mail: nmalavas@ias.u-psud.fr
}

Received 6 July 2021 / Accepted 9 November 2021

\begin{abstract}
Filaments and clusters of the cosmic web have an impact on the properties of galaxies. They switch off their star-formation, contribute to the build-up of their stellar mass, and affect the acquisition of their angular momentum. We make use of the IllustrisTNG simulation, coupled with the DisPerSE cosmic web extraction algorithm, to test which galaxy property is most affected by the cosmic web and, conversely, to assess the differential impact of the various cosmic web features on a given galaxy property. Our aim is to use this information to better understand galaxy evolution and to identify on which galaxy property future efforts should focus to detect the cosmic web from the galaxy distribution. We provide a comprehensive analysis of the relation between galaxy properties and cosmic web features. We also perform extensive tests in which we try to separate the effect of local overdensities of galaxies on their properties from the effect of the large-scale structure environment. Our results show that star formation shows the strongest variation with distance from the cosmic web features, but it also shows the strongest relation to the local environment of galaxies. On the other hand, the direction of the angular momentum of galaxies shows the weakest trends with distance from cosmic web features while also being more independent from the local environment of galaxies. We conclude that the direction of the angular momentum of galaxies and its use to improve our detection of the cosmic web features could be the focus of future studies that will benefit from larger statistical samples.
\end{abstract}

Key words. large-scale structure of Universe - galaxies: clusters: general - galaxies: statistics - galaxies: evolution methods: data analysis - galaxies: formation

\section{Introduction}

Galaxies inhabit a complex network of structures that is called the large-scale structure of the Universe (LSS) or the cosmic web (de Lapparent et al. 1986; Bond et al. 1996; Aragón-Calvo et al. 2010). The cosmic web formed through the gravitational collapse of initial fluctuations of the density field (Zel'dovich $1970 a, b)$ with matter that departed from under-dense regions (which became voids) and collapsed to form walls (twodimensional planar structures that surround voids), filaments (one-dimensional linear structures found at the intersection of walls), and finally flowed inside the filaments to reach clusters that are found at the intersection of filaments. Throughout the duration of this process of structure formation, galaxies evolve while they flow within the cosmic web and experience strong changes in their environment.

It is now well assessed that the cluster environment affects galaxy properties such as their mass, star formation rate (SFR), colours, and morphology (Dressler 1980, 1986; for reviews, see e.g. Boselli \& Gavazzi 2006, 2014). Galaxies in clusters generally have a more elliptical morphology, redder colours, higher mass, and lower SFR than galaxies in less dense environments. With the advent of wide-area spectroscopic surveys, our ability to explore the filaments of the cosmic web and their impact on galaxy properties has increased. Analysing data from the Sloan Digital Sky Survey (SDSS, Abazajian et al. 2009; Alam et al. 2015), the VIMOS Public Extragalctic Redshift Survey Multi- $\lambda$ Survey (VIPERS-MLS, Moutard et al. 2016a,b; Scodeggio et al. 2018), COSMOS2015
(Laigle et al. 2016), the Galaxy And Mass Assembly survey (GAMA, Driver et al. 2009), and the WISExSuperCOSMOS survey (WISExSCOS, Bilicki et al. 2016), Alpaslan et al. (2016), Kuutma et al. (2017), Chen et al. (2017), Malavasi et al. (2017a), Laigle et al. (2018), Kraljic et al. (2018), Bonjean et al. (2020), and Rost et al. (2020) found that massive galaxies and passive galaxies are located closer to the spine of filaments. This effect was also reported by Salerno et al. (2020), who also distinguished between galaxies that accrete onto clusters isotropically and those that follow the filament direction. The authors found that the quenching of galaxies is stronger for galaxies that arrive at clusters by following filaments than for those that accrete onto clusters isotropically (see also e.g. Gouin et al. 2020). Similar trends were also observed in numerical simulations (e.g. by Laigle et al. 2018; Kraljic et al. 2019 using the Horizon-AGN simulation; Dubois et al. 2014).

The features of the cosmic web (clusters and filaments) thus both affect the galaxy properties. In the case of star formation, interaction with the intra-cluster medium (e.g. ram pressure stripping) or tidal interactions with the cluster potential are known to be quenching mechanisms in clusters (see e.g. De Lucia 2007). With respect to filaments, Song et al. (2021) also invoked an inefficient transfer of gas from the outer parts of the haloes to the inner parts as a possible way to prevent star formation activity. This inefficient transfer is caused by the alignment of the vorticity of the gas flow in filaments that accretes high-angular momentum gas onto the haloes. The interactions of galaxies with clusters and filaments could also explain the quenching of the SFR by the disconnection of the galaxies from 
the primordial filaments that supply cold gas to sustain SFR (an event called 'cosmic web detachment'; Aragon Calvo et al. 2019, which would essentially produce quenching by starvation). The global combination of all these quenching mechanisms should produce trends in the galaxy SFR to decrease with the distance to filaments and clusters.

When the properties of galaxies are analysed as a function of their position with respect to the filaments of the cosmic web, a further effect can be extracted from the data regarding their angular momentum (i.e. their spin). According to the tidal torque theory (Peebles 1969; Doroshkevich 1970; White 1984; Catelan \& Theuns 1996; Crittenden et al. 2001), dark matter haloes that host galaxies acquire angular momentum due to the torque that results from a misalignment of their inertia tensor and the external tidal field. Although tidal torque theory generally predicts the amplitude and direction of the angular momentum of haloes acquired throughout their linear evolution to a fair degree of accuracy, later non-linear effects can significantly modify them (Porciani et al. 2002; Dekel et al. 2001; Dutton \& van den Bosch 2012). The tidal torque theory was later expanded to take the fact into account that the filaments and nodes of the cosmic web provide and are shaped by the tidal field that defines the direction of the angular momentum of haloes (constrained tidal torque theory; Codis et al. 2015; Laigle et al. 2015). This context also explains (in terms of the merging of haloes; Bett \& Frenk 2012; Welker et al. 2014, or smooth secondary accretion; Laigle et al. 2015; Ganeshaiah Veena et al. 2018) the change in spin direction that is due to the non-linear halo evolution spent in the filaments and nodes of the cosmic web. According to constrained tidal torque theory and taking into account subsequent halo evolution, haloes are formed with a spin parallel to the filaments of the cosmic web (in particular, low-mass haloes), which changes to perpendicular with increasing halo mass (e.g. Aragón-Calvo et al. 2007; Codis et al. 2012; Hahn et al. 2007a,b) and with the flowing of haloes along the filaments throughout cosmic time (see e.g. Ganeshaiah Veena et al. 2021; Wang \& Kang 2017; Codis et al. 2012, but also Trowland et al. 2013). For this reason, we could expect that a larger fraction of haloes have a spin perpendicular to filaments close to clusters as the endpoint of this evolutionary process. At the same time, the spin could also become randomly oriented with respect to the filaments in clusters because these are essentially regions where the flows from multiple filaments intersect.

The result of this process is a mass transition that is visible in the distribution of the angles between the spin of the haloes and the direction of the closest filament. The spin of low-mass haloes is in this case aligned with the filaments, and that of high-mass haloes is perpendicular. Several works have explicitly tried to bracket this mass transition in simulated and observed data. In particular, for example, Ganeshaiah Veena et al. (2018) explored the alignment of dark matter haloes with respect to filaments in the Planck-Millennium high-resolution $N$-body simulation (McCullagh et al. 2017; Baugh et al. 2019). They reported a transition mass for the spin of the whole halo at $M_{\mathrm{DM}}=5 \times$ $10^{11} M_{\odot}$. Similarly, analysing dark matter haloes in the Horizon $4 \pi N$-body simulation (Teyssier et al. 2009), Codis et al. (2012) detected a higher transition mass $\left(M_{\mathrm{DM}}=5 \times 10^{12} M_{\odot}\right)$. These results are consistent with Kraljic et al. (2020), who detected a transition mass of $M_{\mathrm{DM}}=10^{12} M_{\odot}$ in the Simba simulation (Davé et al. 2019). When galaxies are considered instead of haloes, the trends become weaker (also because a misalignment between the spin of galaxies and the haloes in which they are embedded is possible), but are generally maintained (see e.g.
Codis et al. 2018; Ganeshaiah Veena et al. 2018; Kraljic et al. 2020; Hahn et al. 2010, but see also Ganeshaiah Veena et al. 2019). For example, Ganeshaiah Veena et al. (2018) found that the transition mass from aligned to perpendicular spin with respect to filaments becomes lower when the inner regions of haloes are considered. In actual observations, compatible transition masses are detected for galaxies. For example, Welker et al. (2020) analysed the spin alignment of galaxies in the SydneyAustralian Astronomical Observatory (AAO) Multi-object Integral field spectrograph (SAMI galaxy survey; Croom et al. 2012; Bryant et al. 2015) with respect to filaments detected in GAMA. They reported a transition mass between aligned and perpendicular in the range $M^{*}=10^{10.4} \div 10^{10.9} M_{\odot}$. On the other hand, Krolewski et al. (2019) analysed the spin alignment in the Mapping nearby Galaxies at Apache point survey (MaNGA, Bundy et al. 2015) and did not detect any signal, neither for the total population of galaxies, nor when they divided the total population by mass. However, when they performed the same analysis in the Illustris-1 (Vogelsberger et al. 2014; Nelson et al. 2015) and MassiveBlack-II simulations (Khandai et al. 2015), they detected a transition mass at $M^{*} \sim 10^{10} M_{\odot}$, which is in line with what was found also by Kraljic et al. (2020). When kinematic information about the galaxies is not available, the shape can be used as a proxy to infer the direction of the angular momentum. Trujillo et al. (2006), Paz et al. (2008), Zhang et al. (2013), Tempel et al. (2013), Tempel \& Libeskind (2013), Pahwa et al. (2016), Chen et al. (2019), and Wang et al. (2020), among others, analysed shape alignments in the Sloan Digital Sky Survey (SDSS, York et al. 2000) and in the 2MASS Redshift Survey (2MRS, Huchra et al. 2012). They reported a different degree of alignment according to the mass and morphology of galaxies, while Chen et al. (2015) explored the alignment in the MassiveBlack-II simulation (Khandai et al. 2015).

The SFR and the angle between the spin of galaxies and the direction of the closest filament are also expected to depend on the local environment in which galaxies are embedded. In the case of the SFR, mergers and high-speed interactions between galaxies (harassment), which occur in clusters and in filaments, can contribute to the quenching process (as discussed e.g. in Moutard et al. 2018, 2020). Mergers experienced by galaxies while flowing in the filaments towards the clusters are also invoked as a reason for a transition of the spin direction from aligned to perpendicular to the closest filament (Bett \& Frenk 2012; Welker et al. 2014). The result of this should be trends in the SFR of galaxies to decrease in high-density environments and the spin to become more perpendicular in high-density environments.

A complementary approach to studying galaxy properties in relation to the environment is to use known relations between the distances of galaxies to structures and their observables to better detect and identify features of the cosmic web. In particular, algorithms have been developed to better detect galaxy clusters using the relations between galaxy properties and the environment, such as the redMaPPer approach (Rykoff et al. 2014), which makes use of the defined red sequence in clusters (i.e. the fact that galaxies are redder in denser environments) to improve the detection of galaxy clusters from galaxy surveys. Another example is the work by Rong et al. (2016), which makes use of the average alignment of galaxies to improve the detection of filaments around the Coma cluster (see also e.g. Pandya et al. 2019 , for a possible use of galaxy alignment to detect the cosmic web at high redshift).

The goal of this work is to explore and consolidate these relations by providing a comprehensive view of the trends of 
galaxy properties with respect to the various features of the cosmic web. Our aim is to explore how the SFR, mass, and direction of the angular momentum of galaxies vary with respect to the distance to the nodes and filaments of the cosmic web. By analysing which property varies most strongly with respect to a given feature of the cosmic web, we aim to provide a useful indication of which galaxy observable should be targeted by future galaxy surveys with the aim of better detecting the elusive filaments of the cosmic web. To achieve this goal, we make use of the cosmic web as detected by Galárraga-Espinosa et al. (2020) using the Discrete Persistent Structure Extractor algorithm (DisPerSE, Sousbie 2011; Sousbie et al. 2011) in the IllustrisTNG simulation subhalo catalogue (Nelson et al. 2019).

This paper is organised as follows: we describe the simulation and the algorithm for detecting the cosmic web that we use in Sect. 2. We describe the configuration of distances from the cosmic web features that we consider in Sect. 3.1 and the properties of galaxies that we follow in Sect. 3.2. General results are described in Sect. 4, with further considerations in Sect. 4.5. In Sect. 5 we summarise our results and draw our conclusions. Throughout this paper we use a Planck Collaboration XIII (2016) cosmology with $\Omega_{\Lambda}=0.6911, \Omega_{\mathrm{m}}=0.3089$, and $h=H_{0} / 100=0.6774 \mathrm{~km} \mathrm{~s}^{-1} \mathrm{Mpc}^{-1}$.

\section{Data and method}

We exploited the IllustrisTNG cosmological simulation (Naiman et al. 2018; Pillepich et al. 2018; Nelson et al. 2018, 2019; Marinacci et al. 2018; Springel et al. 2018). It has been performed with the moving-mesh code AREPO (Springel 2010), and it follows the evolution of dark matter, gas, and stars to $z=0$, implementing a Planck Collaboration XIII (2016) cosmology. We used the $z=0$ snapshot of the TNG300-1 box, with a side of $\sim 300 \mathrm{Mpc}$ and $2500^{3}$ dark matter particles for a resolution of $\sim 4 \times 10^{7} M_{\odot} h^{-1}$. Galaxies in this simulation are identified with subhaloes detected by the SUBFIND algorithm (Springel et al. 2001; Dolag et al. 2009). We followed the same selection as Galárraga-Espinosa et al. (2020) (based on Nelson et al. 2019): We discarded all subhaloes flagged by the IllustrisTNG as not reliable (SubhaloFlag $=0$ ) and we applied a cut in stellar mass (SubhaloMassType for star particles) between $10^{9} \leq M^{*} / M_{\odot}<10^{12}$. The final number of subhaloes in our sample is 275818 . Throughout the remainder of the paper, we use the terms subhaloes and galaxies interchangeably.

The filaments of the cosmic web in the simulation volume have been detected by Galárraga-Espinosa et al. (2020) using DisPerSE (Sousbie 2011; Sousbie et al. 2011). DisPerSE identifies filaments from the galaxy distribution by measuring of the gradient of the density field. In our case, the density field was measured through the Delaunay Tessellation Field Estimator (DTFE, Schaap \& van de Weygaert 2000; van de Weygaert \& Schaap 2009), which we applied to the subhaloes selected above to mimic the galaxy distribution ${ }^{1}$. The density field at the position of a considered galaxy was then smoothed by averaging it with the value measured for all galaxies that share an edge of the tetrahedrons of the Delaunay tessellation with this galaxy. When DisPerSE is applied to this density field, it identifies critical points, that is, points at which the

\footnotetext{
1 While it is true that applying DisPerSE to the galaxy distribution provides a different skeleton than the true underlying skeleton that would be obtained by running the algorithm directly on the dark matter particle distribution, characterising the differences between the two is beyond the goal of this work. We refer to Laigle et al. (2018) for an example of such an analysis.
}

gradient is zero (maxima, minima, and saddles). Maxima and saddles are connected with filaments of the cosmic web, which follow lines of constant gradient in the density field. Each filament is composed of small segments that have the size of the edges of the tetrahedrons of the Delaunay tessellation at each position in space. A persistence cut to $3 \sigma$ was applied to remove spurious filaments and critical points caused by the Poisson noise of the density distribution. The ensemble of filaments (and critical points) thus constructed (called skeleton) is then smoothed by averaging the positions of the extrema of each small segment with the positions of the extrema of the two contiguous segments (but keeping the positions of maxima and saddles at the extrema of the filaments unchanged). The skeleton was then broken down and artificial critical points (called bifurcations) were inserted at the positions at which several filaments cross. This was done to take into account the way in which DisPerSE topologically defines filaments: as consistently connecting maxima to saddles. This would cause some filaments to perfectly overlap for part of their path (see Fig. 2 of Galárraga-Espinosa et al. 2020) from the same maximum to diffrent saddles. Bifurcations inserted at the point at which filaments separate to reach separate saddles after having shared a consistent portion of their path solve this problem and avoid duplicating what should be a single portion of a filament. In total, there were 2999 maxima, 4037 bifurcations, and 15220 filaments in the simulation volume. We define filaments in a slightly different way than Galárraga-Espinosa et al. (2020), who defined them as structures detected by DisPerSE that consistently connect maxima to saddles. We defined as filaments any structure detected by DisPerSE that connected several critical points, regardless of their type (e.g. maximumsaddle, maximum-bifurcation, or bifurcation-saddle). We chose this approach in order to be more consistent with observations, where galaxies can be found inside filaments that are connected to dense clusters (better represented by the maxima of the density field in the DisPerSE formalism) and unresolved groups (better represented by bifurcations). In the following we refer to maxima and bifurcations generically as 'nodes'. This word is used in an astrophysical sense (meaning: peaks in the density field) rather than in a topological one (in a topological context, maxima and bifurcations have different definitions).

\section{Analysis}

\subsection{Galaxy distances from the features of the cosmic web}

In this section and the next we present our analysis of the IllustrisTNG simulated data, namely the measurement of the distances of the subhaloes from the components of the cosmic web and the extraction of the various galaxy properties on which we are going to focus in the rest of the paper.

We chose three distances of cosmic web elements that we were able to relate to the various evolutionary paths that a galaxy can take while flowing in the LSS. In particular, when considering variations in galaxy properties as a function of the distance to the axis of the filaments, we can connect recovered trends to the process of galaxies that are infalling onto filaments from within walls. This process is then followed by galaxies that flow inside filaments to reach clusters, which corresponds to a variation in the galaxies' distance from the nodes following the filaments. On the other hand, galaxies can also directly isotropically infall onto clusters. In our case, this would correspond to a variation in the radial distance of the galaxies from the nodes of the cosmic web. We therefore chose the following distances from the cosmic web elements. 
The distance from a galaxy to the axis of the closest filament $\left(d_{\text {fil }}\right)$ is the distance from each galaxy to the midpoint of the closest segment belonging to a filament. The distance from a galaxy to the closest node $\left(d_{\mathrm{CP}}\right)$ is the Euclidean distance from a galaxy to the closest maximum or bifurcation. This distance was computed only for galaxies that have $d_{\text {fil }}>1 \mathrm{Mpc}$ because these galaxies can be considered to lie outside the core of the filaments, based on the density profile for filaments derived by Galárraga-Espinosa et al. (2020). For the distance from a galaxy to the node that is connected to the closest filament following the filaments $\left(d_{\text {skel }}\right)$, we considered for each galaxy the closest filament, and we considered the distance from the point of the filament closest to the galaxy (i.e. the projection of the galaxy position on the filament) to one of the two critical points connected to that filament. The critical point was consistently chosen as the densest of the two: when a filament connected a maximum and a bifurcation or saddle, we chose the maximum. When a filament connected a bifurcation and a saddle, we chose the bifurcation. Finally, when a filament connected two bifurcartions or two saddles, we chose the densest of the considered critical points. This quantity was only computed for galaxies that were located inside the core of the filaments (i.e. having $d_{\mathrm{fil}}<1 \mathrm{Mpc}$ ).

A value of $1 \mathrm{Mpc}$ for the size of the filament core is roughly four times the best-fit scale radius for the generalised Navarro, Frenk, and White profile (Hernquist 1990; Navarro et al. 1997; Nagai et al. 2007; Arnaud et al. 2010) found by Galárraga-Espinosa et al. (2020, see their Table 5). We therefore considered galaxies with $d_{\text {fil }}>1$ to be situated well outside the filament core. However, this value is significantly lower than the value of $27 \mathrm{Mpc}$ mentioned by Galárraga-Espinosa et al. (2020) as an extreme limit for the filaments density profile. We chose a threshold of $d_{\mathrm{fil}}=1 \mathrm{Mpc}$ as a compromise between the best-fit scale radius and the extreme limit of filaments derived in Galárraga-Espinosa et al. (2020) to avoid being affected by too small number counts in either the in-filament or the out-filament sample, which may have an impact on our conclusions. We also tested what happens to the distributions of galaxy properties as a function of distance from the cosmic web features (discussed in Sect. 4) when we considered a threshold distance to identify galaxies inside or outside filaments of $d_{\text {fil }}=0.25,0.5,1,10$, and $27 \mathrm{Mpc}$. Our conclusions do not change, regardless of the chosen threshold.

A schematic view of the considered distances from the cosmic web features is given in Fig. 1. In the remainder of this paper, we refer to the nodes of the cosmic web (maxima and bifurcations) and clusters interchangeably. However, we stress here that it is hard to find a direct correspondence between critical points as identified by DisPerSE and galaxy groups or clusters (see e.g. Fig. 3 of Malavasi et al. 2020a). However, Appendix A shows that considering only the densest critical points in our analysis (i.e. those who are the more likely to match the position of groups and clusters) has only minor effects on our results.

We stress that the node chosen to compute $d_{\mathrm{CP}}$ (the node closest to the considered galaxy) may not be the same as the node considered to measure $d_{\text {skel }}$ (the densest node at the end of the closest filament). For about $30 \%$ of the galaxies in our sample, the two do not match. Moreover, for $22 \%$ of our galaxies, the closest node is not connected to the closest filament. We tested the effect of this discrepancy on our results by eliminating from our sample the galaxies for which the closest node and the densest node at the end of the closest filament used to measure $d_{\text {skel }}$ did not match and re-derived the distributions discussed in Sect. 4. We find no difference in our conclusions when we eliminate these galaxies from our sample.

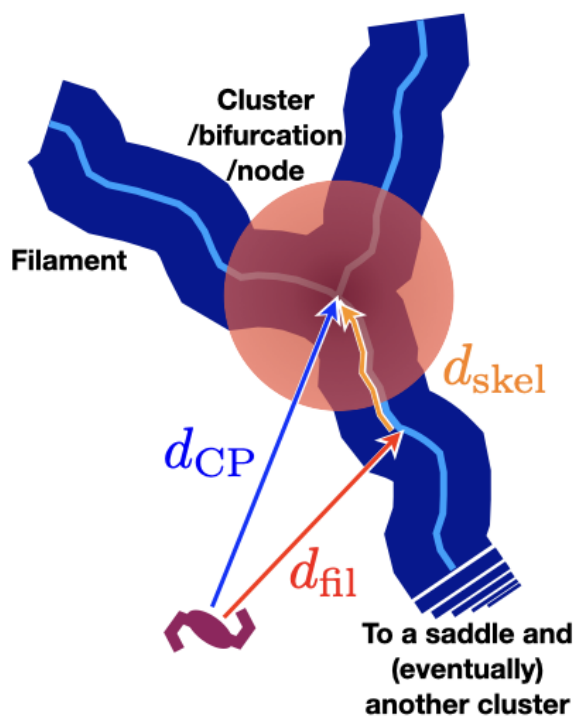

Fig. 1. Schematic representation of the three distances considered in this study: $d_{\text {fil }}, d_{\mathrm{CP}}$, and $d_{\text {skel }} . d_{\text {fil }}$ is the distance from a galaxy to the axis of the closest filament, $d_{\mathrm{CP}}$ is the Euclidean distance from a galaxy to the closest maximum or bifurcation (computed only for galaxies that have $d_{\text {fil }}>1 \mathrm{Mpc}$ ), and $d_{\text {skel }}$ is the distance from a galaxy to the node connected to the closest filament following the filaments (computed only for galaxies that have $d_{\text {fil }}<1 \mathrm{Mpc}$ ).

\subsection{Galaxy properties}

As mentioned in the introduction, for this analysis, we focus on the three main types of galaxy properties that are known to vary in response to the environment of the cosmic web: galaxy stellar mass, observables related to the amount of star formation in galaxies (e.g. SFR or fraction of quenched galaxies), and observables related to the relation between the direction of the galaxies' angular momentum and the direction of the closest filament (e.g. angle between spin and filament direction or fractions of galaxies with a parallel or perpendicular alignment between spin and filaments). Star formation and angular momentum acquisition in galaxies are correlated with mass. In the case of star formation, massive galaxies are known to be forming stars less strongly (see e.g. Strateva et al. 2001; Blanton \& Moustakas 2009; Bolzonella et al. 2010; Pozzetti et al. 2010; Ilbert et al. 2013; Malavasi et al. 2017b, and references therein), while the orientation of the spin with respect to the direction of the large-scale structure changes at the already mentioned transition mass (Ganeshaiah Veena et al. 2018; Codis et al. 2012; Krolewski et al. 2019; Kraljic et al. 2020; Welker et al. 2020). For this reason, in the following we also focus on galaxy quantities that are derived taking the galaxy stellar mass into account (e.g. specific SFR) or we separate the galaxy population into high- and low-mass objects based on the position of the spin transition mass in our data. Several of these galaxy properties are readily available in the IllustrisTNG subhalo catalogue. We explain in the paragraphs below how we extracted them and computed those that were not already present.

\subsubsection{Mass and SFR}

The stellar mass $\left(M^{*}\right)$ in the subhalo catalogue of IllustrisTNG is defined using the SubhaloMassType field for star particles, cut between $10^{9}$ and $10^{12} M_{\odot}$ following Galárraga-Espinosa et al. (2020). The subhalo mass distribution presents a large number 


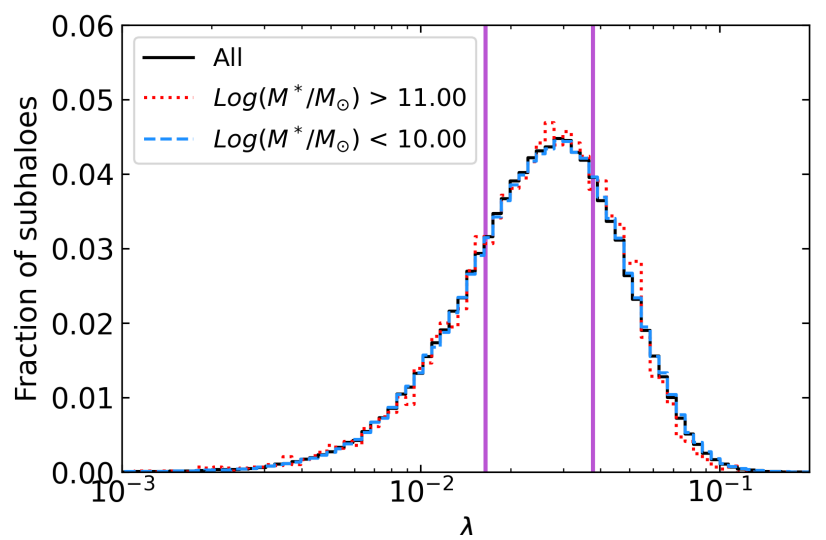

Fig. 2. Distributions of the Bullock parameter $\lambda$ as derived in Eq. (2). The black line refers to the total sample, the dotted red line refers to high-mass galaxies, and the dashed blue line refers to low-mass galaxies. The vertical purple lines are located at the 25 th and 75 th percentiles of the $\lambda$ distribution and represent a qualitative distinction between high-spin parameter and low-spin parameter galaxies.

of low-mass haloes and a progressively decreasing number of high-mass haloes. The break between the two is located at $\sim 3 \times 10^{10} M_{\odot}$. To perform our analysis, we identified two mass regimes, namely high-mass and low-mass subhaloes. We defined high-mass subhaloes as those with $M^{*} \geq 10^{11} M_{\odot}$ and low-mass subhaloes as those with $M^{*} \leq 10^{10} M_{\odot}$. These thresholds were chosen as the limits encompassing the stellar mass range where the transition in galaxy spin alignment from parallel to perpendicular is found in the literature (see e.g. Codis et al. 2018; Welker et al. 2020) and in the IllustrisTNG subhalo sample analysed here (see Sect. 3.2.3). These two values also bracket the position of the knee of the mass function, which represents the typical mass of the average population of galaxies in our sample, and they allow us to explore the extreme tails of the mass distribution. This increases the chance of detecting differences in the behaviour of high- and low-mass galaxies.

The SFR is defined as the sum of the individual SFRs of all gas cells in a given subhalo (in $M_{\odot} \mathrm{yr}^{-1}$ ). Several subhaloes are present with an SFR of zero because no star-forming gas cells were found inside them. We did not eliminate these haloes from the sample, but instead considered them as belonging to the quenched halo population. As an additional quantity to trace star formation in a way that takes galaxy stellar mass into account, we also focused on the specific SFR (sSFR) defined as SSFR = $\mathrm{SFR} / M^{*}$, and we relied on this quantity (rather than on the SFR) to define our quenched galaxy population. We identified quenched galaxies as those with an $\mathrm{SSFR} \leq 10^{-11} \mathrm{yr}^{-1}$ (see e.g. Pozzetti et al. 2010; Davidzon et al. 2016; Donnari et al. 2021, and references therein).

\subsubsection{Spin}

In the IllustrisTNG halo catalogue, the spin per unit mass $(j)$ is defined through the components along each axis of the mass weighted sum of the relative coordinates times the relative velocities of particles. Considering a subhalo and all its member dark matter particles and gas cells of relative coordinates $\boldsymbol{r}_{\mathrm{p}}=\boldsymbol{r}-\boldsymbol{r}_{\mathrm{H}}$ (with $\boldsymbol{r}$ and $\boldsymbol{r}_{\mathrm{H}}$ the absolute coordinates of the considered particle and of the centre of the subhalo to which the particle belongs, respectively) and relative velocities $\boldsymbol{v}_{\mathrm{p}}=\boldsymbol{v}-\boldsymbol{v}_{\mathrm{H}}$ (with $\boldsymbol{v}$ and $\boldsymbol{v}_{\mathrm{H}}$ the absolute velocities of the considered particle and of the centre of the subhalo to which the particle belongs, respectively), the spin is defined as

$\boldsymbol{j}=\frac{\sum_{p \in H} m_{\mathrm{p}} \boldsymbol{r}_{\mathrm{p}} \times \boldsymbol{v}_{\mathrm{p}}}{\sum_{p \in H} m_{\mathrm{p}}}$

(in $\mathrm{Mpc} \frac{\mathrm{km}}{\mathrm{s}}$ ).

The index $p \in H$ indicates all particles of a certain type (and with mass $m_{\mathrm{p}}$ ) that belong to the considered subhalo. Unless otherwise stated, in the following we consider the spin computed using all particles and cells (dark matter, gas, stars, and black holes) belonging to a subhalo (which we refer to as $j_{\text {tot }}$ ). We also computed the spin for individually selected baryonic components such as gas and stars $\left(\boldsymbol{j}_{\text {gas }}, \boldsymbol{j}_{\text {stars }}\right)$ as well as for the total baryonic component of each subhalo $\left(\boldsymbol{j}_{\text {gas }+ \text { stars }}\right)$. Results derived specifically with the spin measured using only certain subhalo components are shown in Appendix B. We made this further check to understand whether the relation between the spin direction and the direction of the filaments that we detect when the full subhalo is considerd is maintained also when components are considered that are more similar to what is targeted with observations of galaxies. The detection of an alignment between filaments and the spin of galaxies is more uncertain in the literature, but Appendix B shows that the trends we detect with the full subhalo are maintained when we consider only the gaseous component. We therefore assume our conclusions to be valid to some extent for galaxies as well.

It is possible to compute the so-called Bullock parameter from the spin (Bullock et al. 2001, also called spin parameter). It is defined as

$\lambda=\frac{j}{\sqrt{2} R_{\mathrm{tot}} V_{\mathrm{c}, \mathrm{tot}}}$,

where $R_{\mathrm{tot}}$ is the radius that includes the entire mass of the subhalo (i.e. the distance from the centre of the subhalo to the farthest particle of any type that is bound to the subhalo), $V_{\mathrm{c}, \text { tot }}=$ $\sqrt{G M_{\mathrm{tot}} / R_{\mathrm{tot}}}$ is the circular velocity at $R_{\mathrm{tot}}$, and $M_{\mathrm{tot}}=\sum_{p \in H} m_{\mathrm{p}}$. Appendix $C$ shows the results obtained when the Bullock parameter is derived using quantities computed at $R_{200}$ instead of $R_{\text {tot }}$.

Figure 2 shows the distribution of the Bullock parameter $\lambda$ for all the subhaloes in the sample. The Bullock parameter distribution for the total subhalo population agrees very well with what was derived for other numerical simulations (see e.g. Ganeshaiah Veena et al. 2018; Hellwing et al. 2021). The distribution is rather smooth, peaks at a value of $\lambda \sim 3 \times 10^{-2}$, and is compact, with short tails at higher and lower values of $\lambda$. This figure also shows the distribution of Bullock parameters for highmass and low-mass galaxies. The two populations do not seem to differ in the distributions of the parameter $\lambda$. In the following, we divide our galaxy population in two samples, namely highspin parameter galaxies (those with $\lambda$ greater than the 75 th percentile of the distribution, i.e. $\lambda=0.038$ ) and low-spin parameter galaxies (those with $\lambda$ lower than the 25 th percentile of the distribution, i.e. $\lambda=0.016$ ). We used the percentiles of the distribution to separate high- and low-spin parameter galaxies to obtain the behaviour of extreme populations in terms of spin parameter amplitude.

\subsubsection{Direction of the angular momentum vector in relation to the LSS}

For each subhalo in the total sample, we measured the angle $\theta$ between the direction of the angular momentum (spin) vector and the local direction of the filament closest to the considered 
subhalo (i.e. the direction of the closest segment as output by DisPerSE). This angle was computed in $3 \mathrm{D}$, and when the spin was not perpendicular to the direction of the filament $(\theta \sim$ $90 \mathrm{deg})$, it could either be aligned $(\theta \sim 0 \mathrm{deg})$ or anti-aligned (i.e. $\theta \sim 180 \mathrm{deg}$ ). We considered alignment and anti-alignment between filaments and spin as the same situation. We therefore limited the angle between spin and filaments to the range $\theta \in[0,90] \mathrm{deg}$. In the following we consider subhaloes to have a spin parallel to the direction of the closest filament if $\theta \leq 30 \mathrm{deg}$, perpendicular if $\theta \geq 60 \mathrm{deg}$, and to have no preferential orientation with respect to the direction of the closest filament if $30 \mathrm{deg} \leq \theta \leq 60 \mathrm{deg}$. We also considered the quantity $\cos (4 \theta)$ to separate the population of subhaloes with a spin either parallel or perpendicular to the filaments and the population of subhaloes with no relation between spin and filament direction. In particular, given its period, the quantity $\cos (4 \theta)$ takes positive values when $\theta \leq 30 \mathrm{deg}$ or $\theta \geq 60 \mathrm{deg}$ and negative values when $30 \mathrm{deg} \leq \theta \leq 60 \mathrm{deg}$. In the following we refer to the case when $\cos (4 \theta)>0$ as subhaloes with an ordered relation between spin and filament direction.

The expected distribution of values of the angle between the direction of the spin and the direction of the closest filament $(\theta)$ for a population of galaxies that is randomly oriented with respect to the surrounding LSS is not uniform in 3D. The expected distribution of $\theta$ values for a population of random galaxies is

$$
P(\theta)=\frac{\sin (\theta)}{2} \text {. }
$$

Figure 3 (top panel) shows that the distribution of angles $\theta$ for the total halo population in the IllustrisTNG box follows the expected distribution for galaxies with random alignments, without particular features regardless of the component used to measure the spin. The distribution of angles varies smoothly between $0 \mathrm{deg}$ and $90 \mathrm{deg}$. The bottom panel of this figure shows the distribution of $\cos (4 \theta)$ for the general galaxy population. The expected distribution of values of $\cos (4 \theta)$ can be derived from Eq. (3) by performing a change of variable to $z=\cos (4 \theta)$. The resulting distribution is

$$
P(z)=\frac{\sin (\arccos (z) / 4)+\sin \left(\arccos (z) / 4+\frac{\pi}{2}\right)}{4 \sqrt{1-z^{2}}} .
$$

The observed distribution follows the expected distribution for a galaxy population with random alignments between the spin of the galaxies and the direction of the closest filament when all the subhaloes in the box are considered for this quantity as well.

Although the distribution of $\theta$ values follows the expected distribution for a sample of randomly oriented subhaloes, a trend for galaxies to be preferentially parallel (perpendicular) to filaments emerges when low-mass (high-mass) galaxies are selected. In the top panel of Fig. 4 we present the ratio of the number of high-mass to low-mass galaxies in three bins of $\theta$, corresponding to the cases of parallel, perpendicular, and no preferential orientation of the spin with respect to the filaments. The general population of the galaxies in the box shows a clear deficit of high-mass galaxies whose spin direction is aligned with the direction of the closest filament. An excess of high-mass galaxies with their spin perpendicular to filaments is also visible. When we split the galaxy population between high-spin parameter and low-spin parameter galaxies using the percentiles of the Bullock parameter distribution, Fig. 4 shows that for high-spin parameter galaxies, the deficit and excess of high-mass galaxies with their
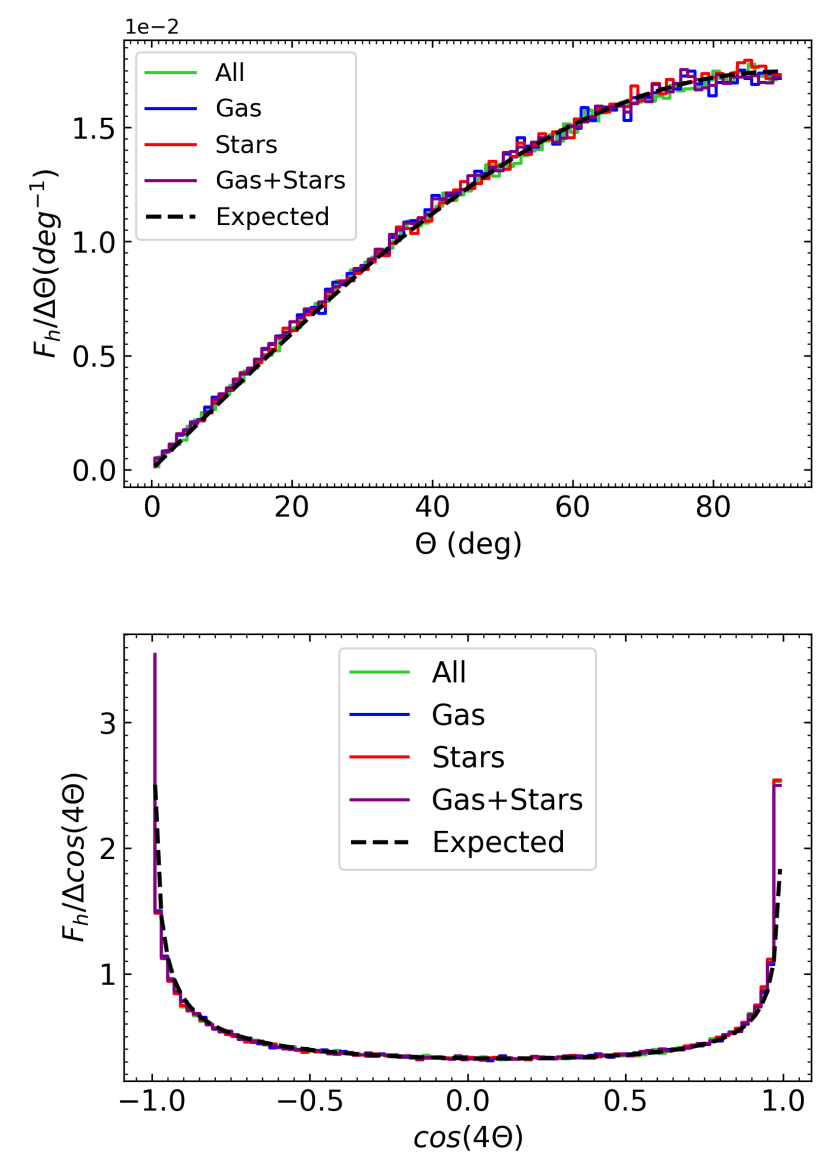

Fig. 3. Distributions of $\theta$ and $\cos (4 \theta)$ for all subhaloes in the box. Top panel: distribution of the values of the angle between the spin of the subhaloes and the direction of the closest filament $(\theta)$. Bottom panel: distribution of values of $\cos (4 \theta)$ for all the subhaloes in the box. In both panels, the solid lines show the measured distribution of values of $\theta$ and $\cos (4 \theta)$ (green: $\boldsymbol{j}_{\text {tot }}$, blue: $\boldsymbol{j}_{\text {gas }}$, red: $\boldsymbol{j}_{\text {stars }}$, and purple: $\boldsymbol{j}_{\text {gas }+ \text { stars }}$ ). The dashed black lines show the expected distribution for a random population of subhaloes (Eq. (3), top panel, and Eq. (4), bottom panel).

spin aligned and perpendicular to the filaments are more significant. In the case of low-spin parameter galaxies, the ratio of the high-mass and the low-mass distribution is consistent with being one.

We explored the mass at which a transition between aligned and perpendicular spin occurs in our data. As stated in the introduction, the result of the evolution of galaxies while they flow in the filaments of the cosmic web is a change in the alignment of their spin with the filaments and an increase in stellar mass. This results in low-mass galaxies retaining a spin parallel to the filaments and high-mass galaxies that spin perpendicular to the filaments. Several works in the literature have tried to bracket the spin transition mass as a means to shed light into the process of galaxy evolution in the cosmic web, but although a broad consensus has been reached, no precise mass value has been obtained.

The bottom panel of Fig. 4 shows the average angle $\langle\theta\rangle$ in a series of increasing mass bins. When all particle types are considered when the spin $\left(\boldsymbol{j}_{\text {tot }}\right)$ is measured, the transition between aligned (i.e. $\langle\theta\rangle<\bar{\theta}$, the expectation value for a random sample of haloes distributed following Eq. (3)) and perpendicular (i.e. $\langle\theta\rangle>\bar{\theta}$ ) occurs at a mass of $\sim 8 \times 10^{10} M_{\odot}$. When only baryonic components are considered, the transition mass decreases to $\sim 4 \times 10^{10} M_{\odot}\left(\right.$ for $\boldsymbol{j}_{\text {gas }}$ and $\boldsymbol{j}_{\text {gas }+ \text { stars }}$ ) and $\sim 2 \times 10^{10} M_{\odot}\left(\right.$ for $\left.\boldsymbol{j}_{\text {stars }}\right)$. 

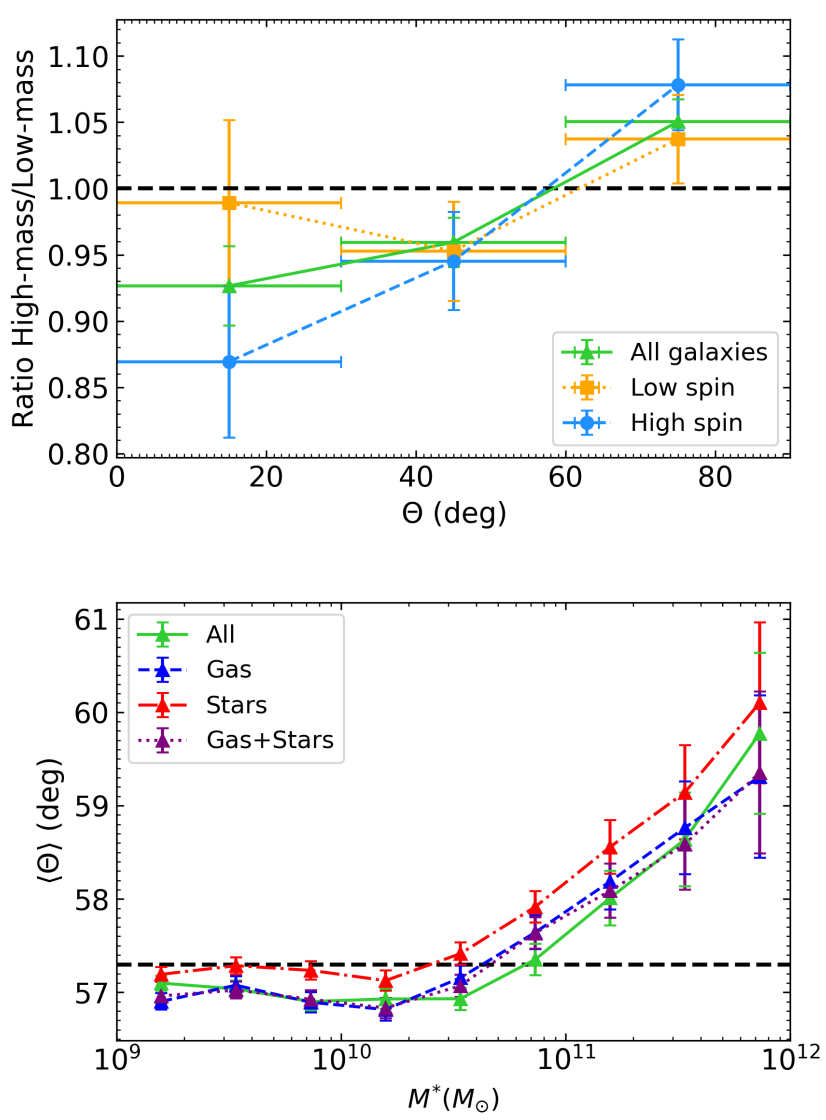

Fig. 4. Detection of a spin alignment transition mass in IllustrisTNG. Top panel: ratio of the distribution of $\theta$ values (binned in three bins corresponding to parallel and perpendicular orientations and to no preferential orientation) as derived for the high-mass and the low-mass galaxies (only $\boldsymbol{j}_{\text {tot }}$ is considered). The green line shows the ratio of high-mass to low-mass $\theta$ distributions for all the galaxies, the dotted orange line shows the ratio for low-spin parameter galaxies, and the dashed cyan line shows the ratio for high-spin parameter galaxies as defined with the percentiles of the Bullock parameter distribution. Bottom panel: average angle $\theta$ computed in bins of stellar mass. The dashed black line is $\bar{\theta}$ given by Eq. (3) for the total subhalo sample. The coloured lines are the distributions for the spin measured with different components (solid green: $\boldsymbol{j}_{\text {tot }}$, dashed blue: $\boldsymbol{j}_{\text {gas }}$, dot-dashed red: $\boldsymbol{j}_{\text {stars }}$, and dotted purple: $\boldsymbol{j}_{\text {gas }+ \text { stars }}$ ).

This range of spin transition masses agrees with that of other works in the literature and represents the first such estimate for the IllustrisTNG simulation. Our choice of limits $10^{10} M_{\odot}$ and $10^{11} M_{\odot}$ to distinguish low- and high-mass galaxies brackets the mass region in which the spin transition occurs. In particular, while the spin transition mass of $M^{*}=10^{11} M_{\odot}$ detected in our sample sets our high-mass limit, the value we chose for a lowmass limit of $M^{*}=10^{10} M_{\odot}$ agrees with what has been reported by Codis et al. (2018) as a spin transition mass. If we limit our galaxy sample to high-spin parameter subhaloes alone, the transition mass value for spin alignment is preserved, while no transition in spin alignment from parallel to perpendicular is visible at any mass for low-spin parameter galaxies (not shown here). This might be an indication that the direction of the angular momentum vector is better defined for galaxies that have a more prominent spin parameter, which leads to a smaller uncertainty in the measurement of the angle between spin and filaments and an increase in the alignment signal that can be extracted for highspin parameter galaxies.

\subsection{Goal of the analysis}

Figure 5 shows in a qualitative way the trends we explore in the remainder of the paper. This figure shows a $25 \mathrm{Mpc}$ thick slice of the simulation box that encompasses the most massive subhalo of the catalogue. In the top panel, the subhaloes in the slice are colour-coded according to the local density contrast as derived directly from the DTFE density $\left(1+\delta_{\rho}=\rho_{\text {DTFE }} /\left\langle\rho_{\text {DTFE }}\right\rangle\right.$, where the average is computed over the full box). Filaments from DisPerSE are overlaid in green, and their path precisely follows the density field, as expected. Critical points identified by DisPerSE (only maxima and bifurcations are shown for the sake of clarity, shown in black in the figure) are found at the intersection of filaments.

The four bottom panels show the same filaments and critical points as the top panel, but different sets of galaxies are represented. In particular, the figure shows how star-forming and quenched galaxies (with the distinction between the two set at $\left.\mathrm{sSFR}=10^{-11} \mathrm{yr}^{-1}\right)$, high-mass and low-mass galaxies (with the distinction set at $M^{*} \geq 10^{11} M_{\odot}$ and $M^{*} \leq 10^{10} M_{\odot}$, respectively), parallel and perpendicular (i.e. $\theta \in[0,30]$ deg and $\theta \in[60,90] \mathrm{deg}$, respectively), and ordered and without a preferential direction (with the distinction between the two being set at $\cos (4 \theta)=0$ ) are distributed with respect to the nodes and the filaments. This figure shows that massive galaxies are very rare and are mostly located in dense regions at the intersection of filaments, tracing the density peaks. On the other hand, low-mass galaxies are more uniformly distributed around filaments. The same is true for star-forming galaxies, while quenched galaxies mostly tend to cluster at nodes. Only a few galaxies lie around the filaments. However, parallel galaxies do not show a particular tendency for clustering around nodes. Rather, they tend to be more uniformly distributed around filaments, highlighting the position of the features of the cosmic web. This is even more evident in the case of ordered galaxies, which clearly delineate the position of high-density and low-density structures of the cosmic web (nodes and filaments). This figure qualitatively shows, however, that different properties of galaxies may trace the various features of the cosmic web. Because galaxy and halo spin direction is mainly initially set by the filaments (constrained tidal torque theory) and is changed by subsequent evolution of the galaxies in the filaments while they flow towards the clusters, we expect the alignment to be stronger in the filament environment, which we therefore expect to be better traced by this galaxy property. On the other hand, other properties such as SFR are strongly affected by the dense and hot gaseous environment of clusters, for instance, and are therefore expected to be a better tracer of nodes. The goal of this work is to test whether these expectations are correct. While the effect of the clusters on the SFR and the mass of galaxies has been extensively tested and recent results started to investigate the effect of filaments on these quantities and the spin alignment of galaxies, we try here to quantify the relative importance of mass, SFR, and spin to characterise the cosmic web. We also determine the impact of clusters and filaments on these observables.

\section{Results}

We derived the distributions of the average stellar mass $\left(M^{*}\right)$, (specific-)SFR, $\theta, \cos (4 \theta)$, and fractions of quenched, ordered, parallel, and perpendicular galaxies $\left(f_{\mathrm{Q}}, f_{\text {Ord }}, f_{\|}\right.$, and $f_{\perp}$, respectively) as a function of $d_{\text {fil }}, d_{\mathrm{CP}}$, and $d_{\text {skel }}$ (Fig. 6). This figure offers a global and comprehensive view of the variation in the various quantities with the considered distances. In the 

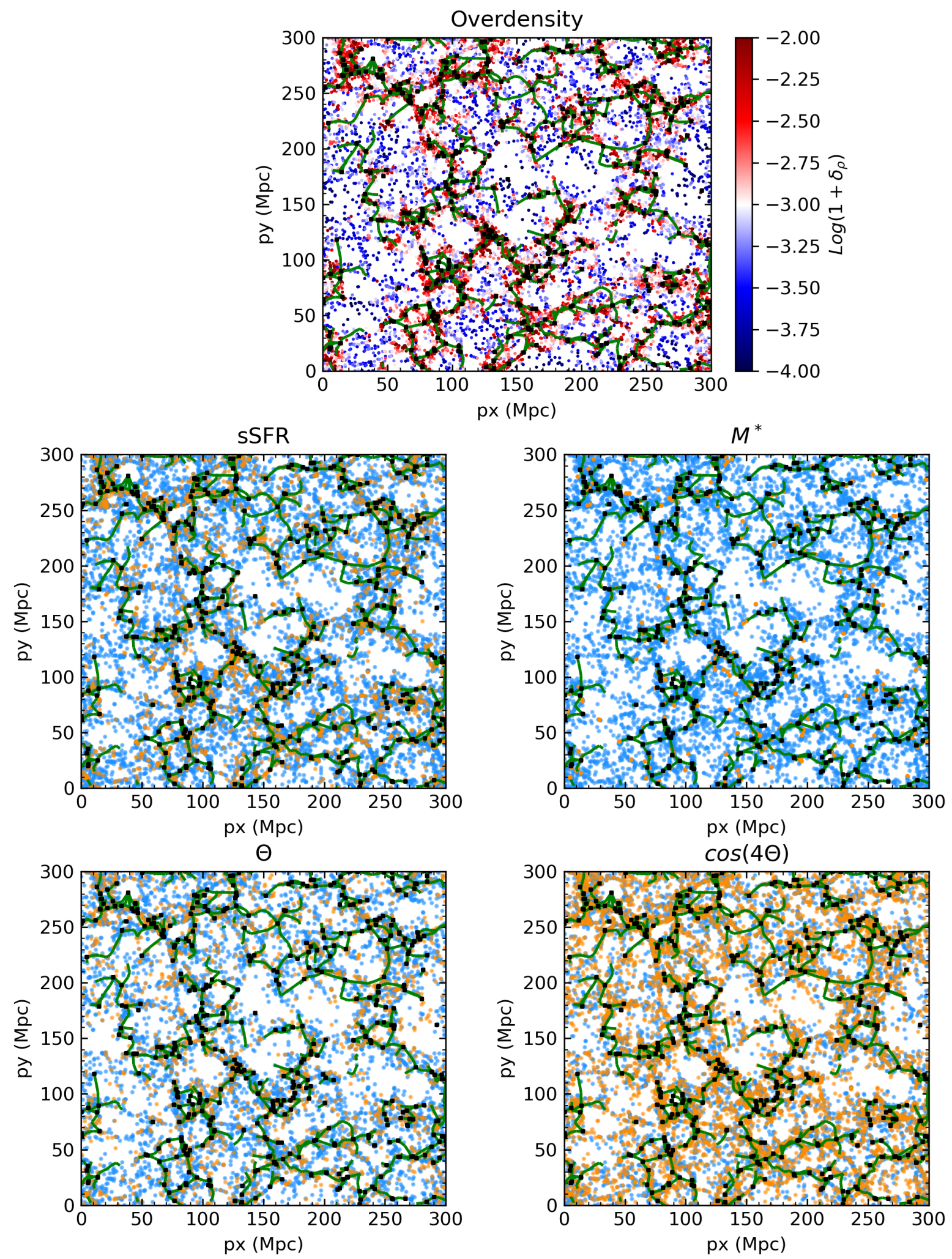

Fig. 5. Galaxy positions with respect to the cosmic web features. This figure shows a slice $25 \mathrm{Mpc}$ thick of the simulation box that encompasses the most massive subhalo of the simulation. In all panels, points represent galaxies, green lines show filaments, and black squares are maxima and bifurcations as derived by DisPerSE. Top panel: subhaloes are colour-coded according to their local density $\left(\log \left(1+\delta_{\rho}\right)\right)$; all subhaloes are shown. In the bottom four panels, subhaloes are colour-coded according to which population they belong: star-forming (light blue) or quenched (orange) in the middle left, low-mass (light blue) or high-mass (orange) in the middle right, perpendicular (light blue) or parallel (orange) in the bottom left, no orientation (light blue) or ordered (orange) in the bottom right. See Sect. 3.2 for the distinction in the various subsets. Top left and bottom right panels: all subhaloes are shown, while in the other panels, subhaloes that do not belong to the considered populations are not shown. 

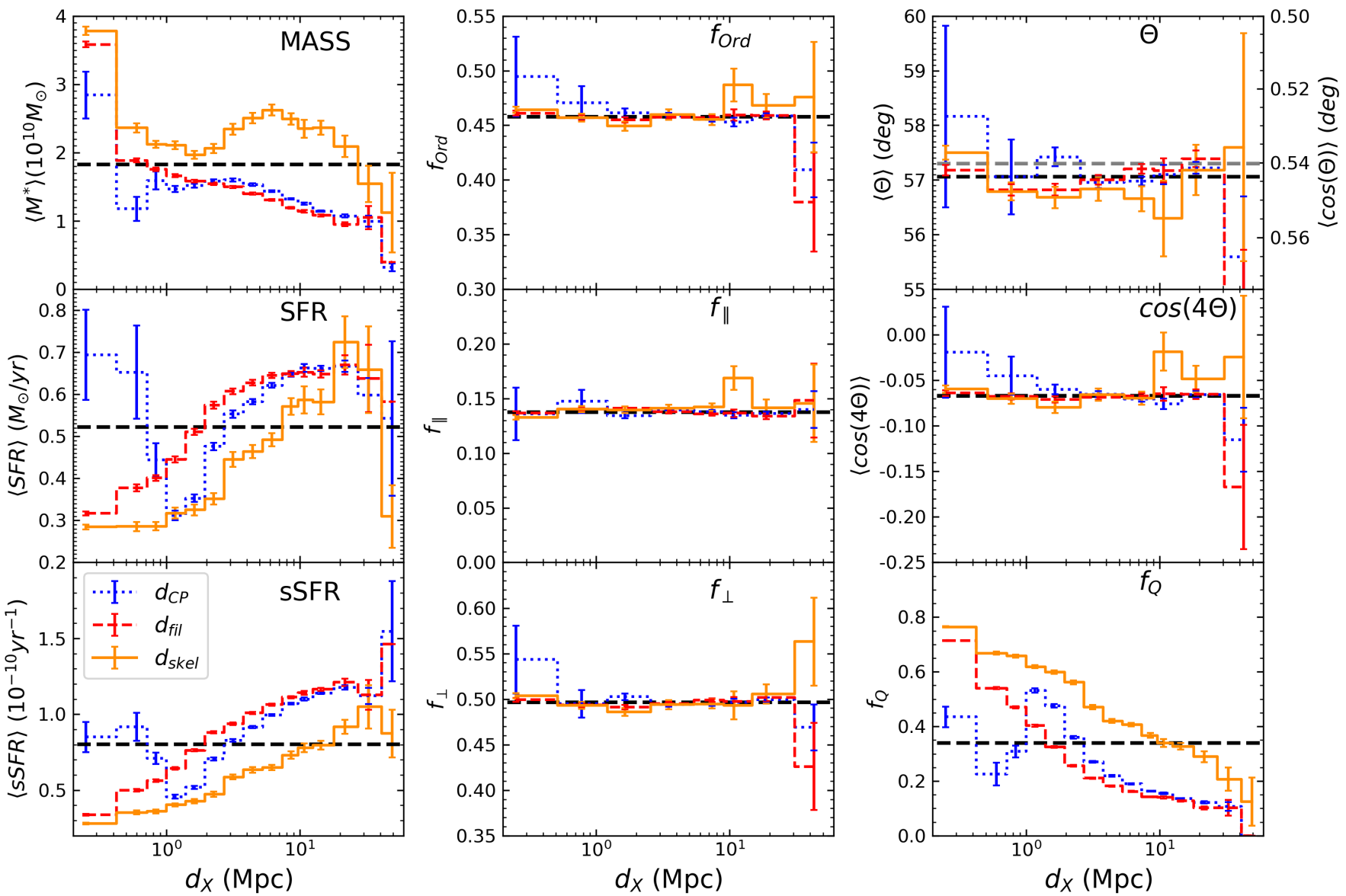

Fig. 6. Distributions of $\left\langle M^{*}\right\rangle,\langle\mathrm{SFR}\rangle,\langle\mathrm{sSFR}\rangle,\langle\theta\rangle,\langle\cos (4 \theta)\rangle$, fraction of quenched galaxies $\left(f_{\mathrm{Q}}\right)$, fraction of ordered galaxies $\left(f_{\text {Ord }}\right)$, fraction of parallel galaxies $\left(f_{\|}\right)$, and fraction of perpendicular galaxies $\left(f_{\perp}\right)$ as a function of the distances from the features of the cosmic web $d_{\mathrm{fil}}(\mathrm{dashed}$ red line in every panel), $d_{\mathrm{CP}}$ (dotted blue line in every panel), and $d_{\text {skel }}$ (solid orange line in every panel). In the case of $d_{\mathrm{CP}}$ and $d_{\text {skel }}$, only galaxies outside of filaments $\left(d_{\mathrm{fil}} \geq 1 \mathrm{Mpc}\right)$ and inside filaments $\left(d_{\mathrm{fil}} \leq 1 \mathrm{Mpc}\right)$ have been considered, respectively. Error bars on the distributions have been computed through bootstrap resampling. The dashed black line in every panel is the average of the considered quantity in the full simulation box. In the top and middle panels of the right column, the grey line is $\bar{\theta}$ and $\overline{\cos (4 \theta)}$, computed given Eqs. (3) and (4) for the total subhalo sample. In this figure, the dashed grey line corresponding to $\overline{\cos (4 \theta)}$ is hidden behind the dashed black line in the same panel. In every panel, the first distance bin we considered extends all the way to 0 for all distances, but it is cut because the $x$-axis in in logarithmic scale. In the $f_{\|}$and $f_{\perp}$ cases, the $y$-axes of the plots cover very different ranges.

following, we describe the trends with distances from the features of the cosmic web for mass and SFR-related galaxy properties and spin-related galaxy properties separately.

\subsection{Mass and SFR-related quantities}

Stellar mass $\left\langle M^{*}\right\rangle,\langle\mathrm{SFR}\rangle,\langle\mathrm{sSFR}\rangle$, and $f_{\mathrm{Q}}$ all vary monotonically with $d_{\text {fil }}, d_{\mathrm{CP}}$, and $d_{\text {skel }}$. $\left\langle M^{*}\right\rangle$ and $f_{\mathrm{Q}}$ decrease farther away from structures, and $\langle\mathrm{SFR}\rangle$ and $\langle\mathrm{sSFR}\rangle$ increase. These trends reflect the fact that more massive galaxies inhabit the inner regions of structures, which are also the places at which galaxy populations experience a higher degree of quenching. A decrease in SFR with decreasing distance from the spine of filaments is also reported in observations (e.g. Bonjean et al. 2020; Kuutma et al. 2017). However, $\left\langle M^{*}\right\rangle$ has a very similar trend with distances, regardless of whether we consider a node or a filament (i.e. with respect to $d_{\mathrm{CP}}$ and $d_{\mathrm{fil}}$ ). The only difference between the curves is for trends with $d_{\text {skel }}$, that is, galaxies inside filaments for which the mass is higher on average, but varies less significantly closer to the nodes following the filamentary structures. The increase in the distribution of $\left\langle M^{*}\right\rangle$ at high $d_{\text {skel }}$ values is due to small number counts in the bins in this regime, due to our choice of a dis- tance of $d_{\text {fil }}=1 \mathrm{Mpc}$ to separate between the populations within and outside the core of the filaments (as $d_{\text {skel }}$ is defined only for subhaloes with $d_{\text {fil }} \leq 1 \mathrm{Mpc}$ ). When a larger distance threshold is chosen, these bins become more populated and the distribution decreases monotonically and becomes closer to the distributions for $d_{\mathrm{CP}}$ and $d_{\mathrm{fil}}$. On the other hand, quantities related to SFR show some degree of difference between the structures, with the three curves for $d_{\mathrm{CP}}, d_{\text {fil }}$, and $d_{\text {skel }}$ being separated. Therefore, structures seem to affect the SFR differently than they do $M^{*}$, which means that SFR can be used to better separate whether galaxies are close to filaments or to nodes. Moreover, for $\langle\mathrm{SFR}\rangle$, the increase seen at small $d_{\mathrm{CP}}$ arises because these bins have low number counts due to our choice of a distance threshold to separate between galaxies in filaments and outside filaments (as $d_{\mathrm{CP}}$ is defined only for subhaloes with $d_{\mathrm{fil}} \geq 1 \mathrm{Mpc}$ ). A lower distance threshold has the effect of making these bins more populated, and the $\langle\mathrm{SFR}\rangle$ distribution increases monotonically with $d_{\mathrm{CP}}$.

\subsection{Spin-related quantities}

Spin-related quantities $\left(\langle\theta\rangle,\langle\cos (4 \theta)\rangle, f_{\text {Ord }}, f_{\|}\right.$, and $\left.f_{\perp}\right)$ show little to no variation with respect to the distances from the various 


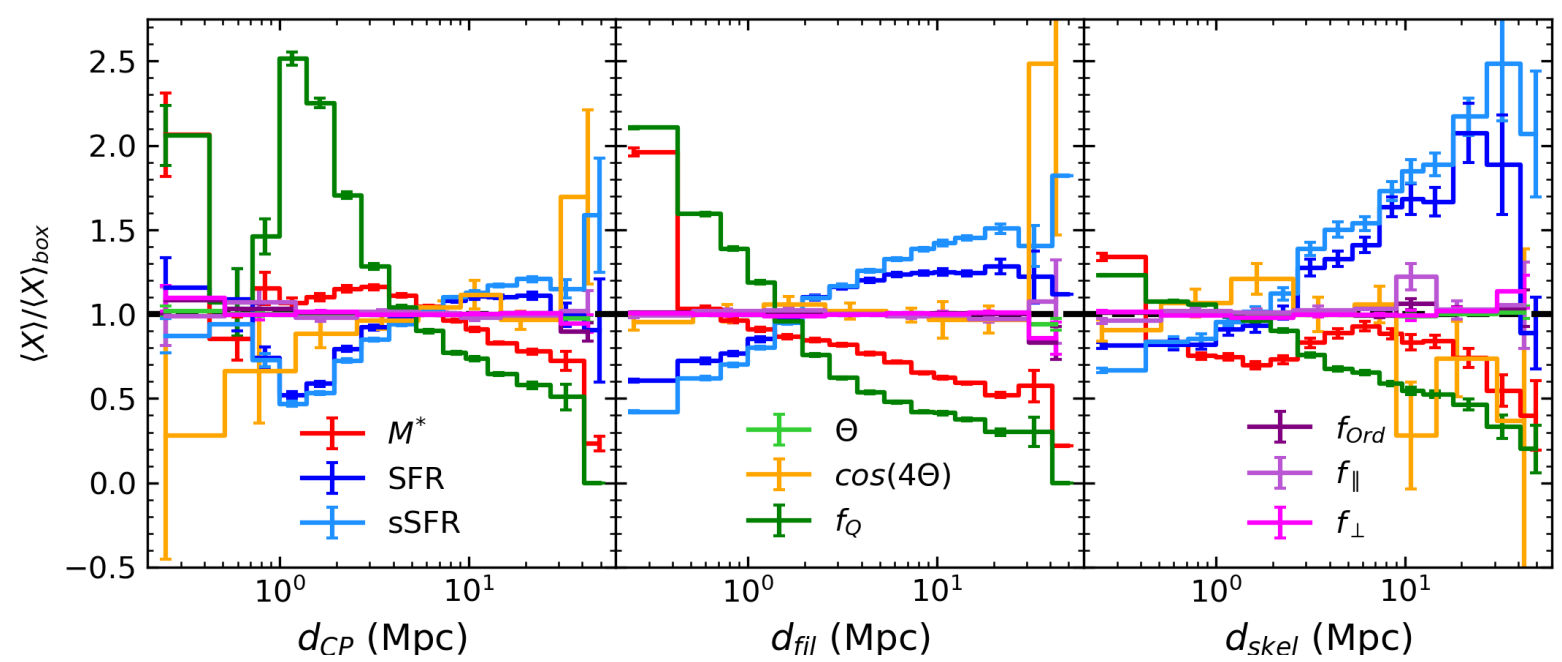

Fig. 7. Normalised distributions (expressed as $\frac{\langle X\rangle}{\langle X\rangle_{\mathrm{box}}}$ ) with respect to distances $d_{\mathrm{CP}}$ (left panel), $d_{\mathrm{fil}}$ (middle panel), and $d_{\mathrm{skel}}$ (right panel). The quantities considered in each panel $(\langle X\rangle)$ are $\left\langle M^{*}\right\rangle$ (red), $\langle\mathrm{SFR}\rangle$ (dark blue), $\langle\mathrm{sSFR}\rangle$ (light blue), $\langle\theta\rangle$ (light green), $\langle\cos (4 \theta)\rangle$ (orange), $f_{\mathrm{Q}}(\mathrm{dark}$ green), $f_{\text {Ord }}$ (dark purple), $f_{\|}$(light purple), and $f_{\perp}$ (magenta). $\langle X\rangle_{\text {box }}$ indicates the average of the quantity taken including all subhaloes in the box (for $d_{\mathrm{fil}}$ ), only those outside filaments (for $d_{\mathrm{CP}}$ ), and only those inside filaments (for $d_{\text {skel }}$ ) over the full simulation volume. Error bars on the distributions have been computed through bootstrap resampling. In every panel, the first distance bin considered extends all the way to zero for all distances, but it is cut because the $x$-axis is in logarithmic scale.

structures. The various distributions are often rather flat and overlap. The only exception is the case of $\langle\theta\rangle$, which shows a flat distribution for $d_{\text {fil }}$, but not for $d_{\text {skel }}$. In this latter case, the value of $\langle\theta\rangle$ decreases with distance from nodes following the filaments. In the case of $d_{\mathrm{CP}}$, there is a trend for a larger fraction of galaxies with their spin perpendicular to the filaments to be present (visible in an increased value for $\langle\theta\rangle,\langle\cos (4 \theta)\rangle, f_{\text {Ord }}$, and $f_{\perp}$ ) at low values of $d_{\mathrm{CP}}$, but large error bars prevent us from confirming this. These results fit in the theoretical framework outlined by constrained tidal torque theory (Codis et al. 2015). As stated in the introduction, constrained tidal torque theory predicts the alignment of the spin of haloes with the filaments of the cosmic web. This alignment is subsequently changed to perpendicular by the further non-linear evolution of the filaments while they flow within the filaments. This can be due, for example, to the smooth accretion of vorticity-rich gas onto haloes that happen to be larger than a given vorticity quadrant of the filaments (Laigle et al. 2015; see also Ganeshaiah Veena et al. 2018: accreting haloes are generally embedded in thinner filaments, and the subsequent accretion contributes to the spin becoming perpendicular to the filaments). As non-linear processes change halo spin while they flow within filaments towards clusters (Wang \& Kang 2017), we expect a larger fraction of galaxies with spin parallel to the filaments far away from the clusters, which progressively reduces towards the clusters. In clusters, we either expect a random orientation of spin (as these are multi-flow regions) or a preferentially perpendicular orientation of spin with respect to filaments (which our results seem to indicate).

\subsection{Variation in galaxy properties with respect to a given cosmic web distance}

In order to compare how a given structure or infall path (nodes or filaments) is traced by each quantity, we normalised the distributions shown in Fig. 6 to the average of each considered quantity, computed in the full extent of the box. In the case of average quantities such as $\left\langle M^{*}\right\rangle,\langle\mathrm{SFR}\rangle,\langle\mathrm{sSFR}\rangle,\langle\theta\rangle$, and $\langle\cos (4 \theta)\rangle$, we divided the distributions shown in Fig. 6 by the average of the same quantities computed using all the subhaloes in the box. In the case of fraction quantities, that is, $f_{\mathrm{Q}}, f_{\text {Ord }}, f_{\|}$, and $f_{\perp}$, we divided the distributions of Fig. 6 by the fractions of the same quantities computed using all subhaloes in the box. The trends of the quantities shown in the panels of Fig. 6 for $d_{\mathrm{fil}}, d_{\mathrm{CP}}$, and $d_{\text {skel }}$ are shown in Fig. 7.

The normalised distributions show how different galaxy properties trace the same type of structure. In this regard, when $d_{\mathrm{CP}}$ is considered, $f_{\mathrm{Q}}$ seems to be the quantity that shows the greatest variation across the range of considered distances. This remains generally true also for $d_{\text {fil }}$, although in this case, the SFR and sSFR acquire importance as well, especially in tracing large distances from filaments. Finally, these two latter quantities trace $d_{\text {skel }}$ best of all. In the context of spin-related quantities, only $\cos (4 \theta)$ shows a variation comparable to other quantities, at small $d_{\mathrm{CP}}$, although with large error bars.

\subsection{Global versus local environment}

We explored the relation between the global environment of galaxies (i.e. their position with respect to LSS features) and their local environment (i.e. the environmental density in their immediate vicinity, regardless of the type of structures in which they are embedded). In our case, the global environment of galaxies was explored through the distances introduced above $\left(d_{\mathrm{CP}}, d_{\mathrm{fil}}\right.$, and $\left.d_{\text {skel }}\right)$. The local environment was instead codified through the DTFE density value at the position of each galaxy (in $\mathrm{Mpc}^{-3}$ ), which was directly measured by DisPerSE and used to derive the skeleton.

Figure 8 shows the distributions of density values for all galaxies and for those inside and outside of filaments. The distribution for all galaxies shows a peak at low density values (slightly above $10^{-2} \mathrm{Mpc}^{-3}$ ) and decreases slowly, reaching high values of $\sim 10^{3} \mathrm{Mpc}^{-3}$. Selecting only galaxies inside or outside filaments has the effect of restricting the density range considered (the distribution for galaxies inside filaments indeed peaks at higher values $\gtrsim 10^{1} \mathrm{Mpc}^{-3}$, while the distribution for galaxies 


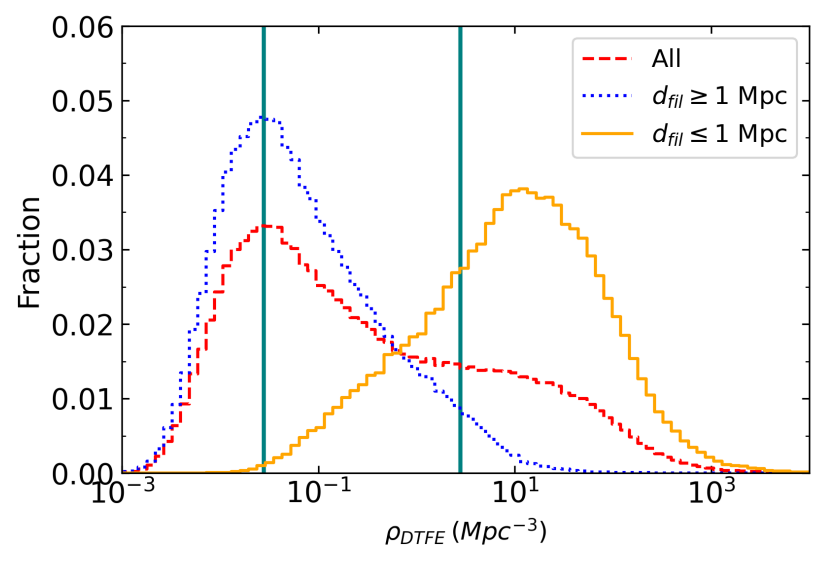

Fig. 8. Distributions of density $\left(\rho_{\text {DTFE }}\right)$ as measured through the DTFE value output by DisPerSE at the position of each galaxy. The dashed red line refers to the total population of galaxies, the dotted blue line to the galaxies outside of filaments, and the solid yellow line to the galaxies inside filaments. The two vertical blue lines represent the 25 th and 75 th percentiles of the total distribution.

outside filaments peaks at $\gtrsim 10^{-2} \mathrm{Mpc}^{-3}$ ). This confirms that galaxies outside filaments are in environments that are locally less dense on average than those of galaxies within filaments. However, the large overlap between the local density distributions of galaxies inside and outside filaments strongly supports the fact that local density alone is not a good criterion for separating the features of the cosmic web and that a topological definition has to be used to detect the filaments. This overlap in local density for galaxies belonging to different cosmic structures poses the question whether the trends that we detect and showed above are due to local density or to the anisotropic properties of the filaments. If galaxies inside and outside filaments can experience the same kind of local environment while belonging to different types of global LSS features (and similarly for galaxies inside clusters or on their outskirts), then it might mean that galaxies outside and inside cosmic web structures can share the same properties if these are driven predominantly by local density.

Figure 9 shows how the average local density $\left\langle\rho_{\text {DTFE }}\right\rangle$ varies as a function of the considered distances from the structures. As expected, the local density decreases monotonically with distance from structures. For galaxies within filaments, the density is consistently higher than in other cases, and it seems to present a milder decrease as well. As different cosmic web features broadly cover different density ranges, any dependence of galaxy properties on local density rather than on their global environment could mimic a trend with distance from the cosmic web features as those shown above. In order to confirm the effect of local density on our conclusions, we performed the following analysis.

If we derive the same distributions as in Fig. 6 separated between high- and low-density galaxies (not shown here), we find that in general, galaxies follow the expected trends in the high- and low-density case, although they are less clearly defined than when the global galaxy population is considered. In general, high-density galaxies are less strongly star-forming and show a higher fraction of quenched systems. High- and lowdensity galaxies both show trends with the various distances from structures, with the (specific-)SFR increasing and the fraction of quenched galaxies decreasing with increasing $d_{\mathrm{CP}}, d_{\mathrm{fil}}$, and $d_{\text {skel }}$. Spin-related quantities do now show any difference between high- and low-density galaxies, and no trends with the

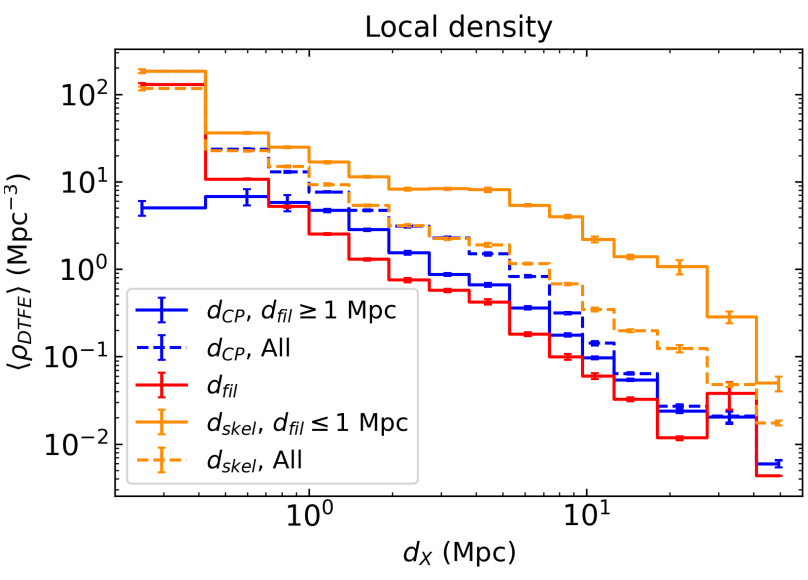

Fig. 9. Distributions of $\left\langle\rho_{\mathrm{DTFE}}\right\rangle$ as a function of the distances from the features of the cosmic web $d_{\text {fil }}$ (red line), $d_{\mathrm{CP}}$ (blue line), and $d_{\text {skel }}$ (orange line). In the case of $d_{\mathrm{CP}}$ and $d_{\text {skel }}$, solid lines refer to galaxies outside of filaments $\left(d_{\mathrm{fil}} \geq 1 \mathrm{Mpc}\right)$ and inside filaments $\left(d_{\mathrm{fil}} \leq 1 \mathrm{Mpc}\right)$, respectively, while dashed lines refer to the total galaxy population. Error bars on the distributions have been computed through bootstrap resampling. In every panel, the first distance bin considered extends all the way to zero for all distances, but it is cut because the $x$-axis is in logarithmic scale.

distances from structures seem to be visible when galaxies are separated according to their local environment. We separated local high- and low-density environments using the 75th and the 25 th percentiles of the local density distribution, respectively (shown in Fig. 8).

Figure 10 shows the dependence of galaxy properties on the local density. As expected, trends with local density are visible for mass and SFR-related quantities. As the density increases, galaxy $M^{*}$ also increases, while SFR and sSFR decrease. Correspondingly, in denser local environments, the fraction of quenched galaxies is higher. On the other hand, no trend with local density can be seen for spin-related quantities. Moreover, the distributions for galaxies inside filaments and outside filaments show no difference, regardless of the quantity considered.

In order to distinguish the effect of local density from the effect of structures, we performed the following test: We divided the galaxy sample into local density bins and shuffled the considered galaxy quantity (mass, SFR, etc.) 1000 times within the density bin while keeping the distances to the LSS features $\left(d_{\mathrm{CP}}\right.$, $\left.d_{\text {fil }}, d_{\text {skel }}\right)$ unchanged. This eliminated the relation between a given quantity and the distances to structures, but kept the relation between the given quantity and local density intact (because the galaxy property values mix among galaxies within the same local density bin). In this way, if galaxies in a given local density bin have different properties than elsewhere (e.g. a lower SFR on average), this will be preserved, while the shuffling eliminates the relation between the given quantity and the distances to the cosmic web features.

The distributions of mass and SFR-related quantities do not change visibly when galaxies are re-shuffled. In these cases, we cannot exclude that the trends that we see are due to the variation in local density in response to the distance to the various structures, rather than to the structures themselves. On the other hand, for spin-related quantities, and in particular, for the number of galaxies whose spin is perpendicular to the filaments, we see variations in the distributions of re-shuffled galaxies, in particular at small distances from nodes for galaxies outside filaments. This is an indication that the spin of galaxies might be a 

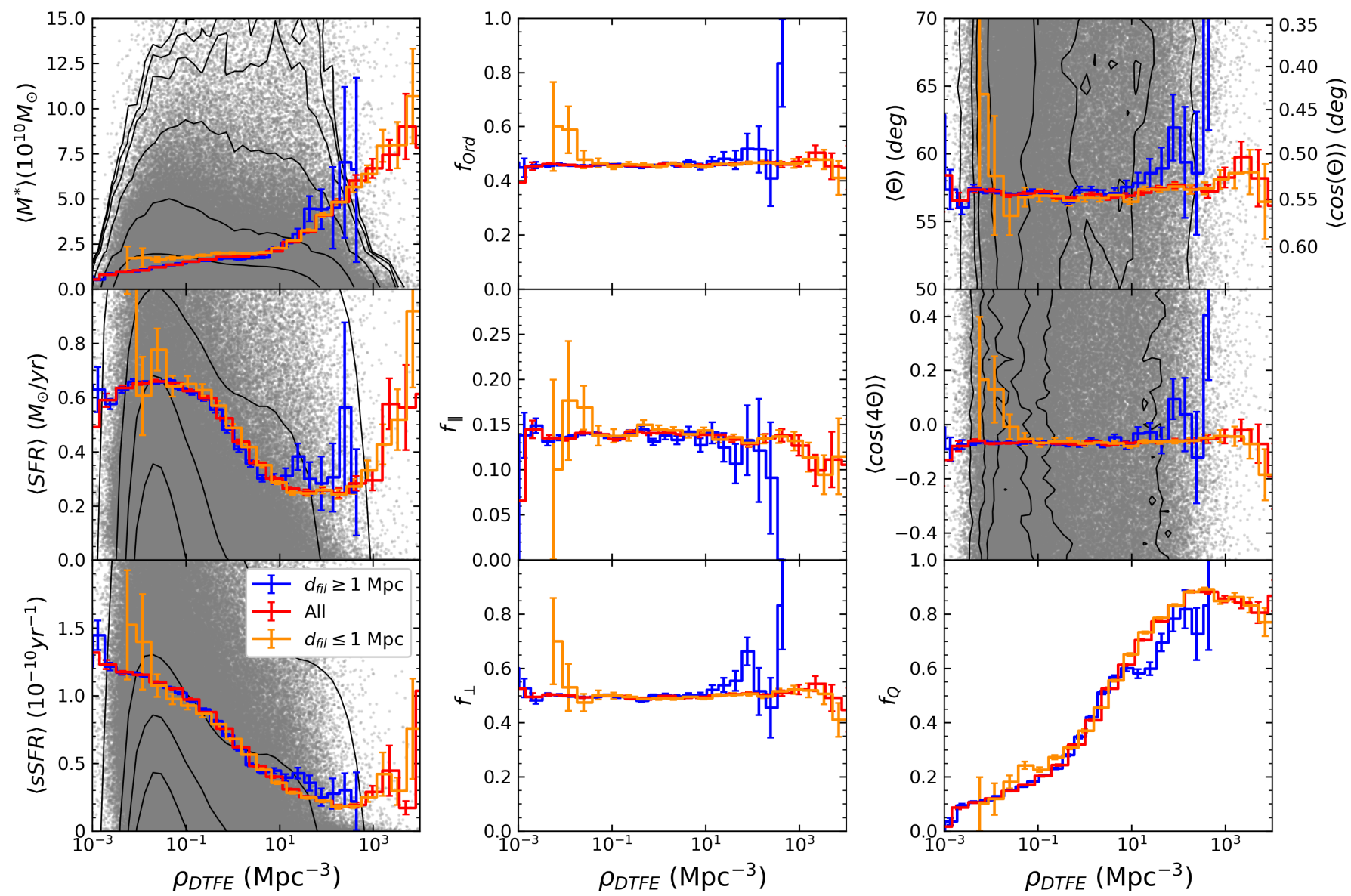

Fig. 10. Distributions of $\left\langle M^{*}\right\rangle,\langle\mathrm{SFR}\rangle,\langle\mathrm{sSFR}\rangle,\langle\theta\rangle,\langle\cos (4 \theta)\rangle$, fraction of quenched galaxies $\left(f_{\mathrm{Q}}\right)$, fraction of ordered galaxies $\left(f_{\text {Ord }}\right)$, fraction of parallel galaxies $\left(f_{\|}\right)$, and fraction of perpendicular galaxies $\left(f_{\perp}\right)$ as a function local density $\left(\rho_{\text {DTFE }}\right)$ for all galaxies (red line in every panel), galaxies inside filaments $\left(d_{\text {fil }} \leq 1 \mathrm{Mpc}\right.$, orange line in every panel), and outside of filaments $\left(d_{\text {fil }} \geq 1 \mathrm{Mpc}\right.$, blue line in every panel). Error bars on the distributions have been computed through bootstrap resampling. Where present, grey points represent the scatter of the total galaxy population in the considered quantity-local density plane (black contours show the shape of the distribution where points saturate). In the $f_{\|}$and $f_{\perp}$ cases, the $y$-axes of the plots cover very different ranges.

way to trace the cosmic web. This way is more independent of local density than other quantities.

We have tried to quantify the difference between the reshuffled and the original distributions by means of the quantity $\frac{H_{\mathrm{O}}-H_{\mathrm{R}}}{\sqrt{\sigma_{\mathrm{O}}^{2}+\sigma_{\mathrm{R}}^{2}}}$, where $H_{\mathrm{O}}$ are the original distributions shown in Fig. 6 , $H_{\mathrm{R}}$ the re-shuffled ones, and $\sigma_{\mathrm{O}}$ and $\sigma_{\mathrm{R}}$ their uncertainties. This quantity highlights any significant change between the original and re-shuffled distributions and is shown in Fig. 11. We find that in the case of $\langle\theta\rangle$, the differences among the distributions are as high as $0.5 \sigma$, while in the case of $\langle\cos (4 \theta)\rangle, f_{\text {Ord }}, f_{\|}$, and $f_{\perp}$ they are in the range of $0.2 \sigma$. Although the differences between the original and re-shuffled distributions of spin-related quantities are not large, they are still detected. In particular, in the case of $\langle\cos (4 \theta)\rangle$ and $f_{\text {Ord }}$ when considered with respect to $d_{\mathrm{CP}}$, the differences are consistently positive, meaning that the re-shuffled distributions have a smaller amplitude than the original ones. This is in sharp contrast with the case of $\langle\mathrm{sSFR}\rangle$, where the differences between the re-shuffled and the original distributions are as low as $10^{-5} \sigma$. This supports the hypothesis that although trends of spin-related quantities with distances from the cosmic web are more difficult to detect, they may be more insensitive to the local density of galaxies.

In the case of $\langle\mathrm{SFR}\rangle$ and $f_{\mathrm{Q}}$, the differences between the distributions are also in the range of $0.5 \sigma$ as for $\langle\theta\rangle$, but they oscil- late around zero without being consistently positive or negative across the range of distances we considered. The largest differences between re-shuffled and original distributions are observed in the case of mass (of about $10 \sigma$ ), but this might be due to the precision with which stellar mass is measured.

\subsection{Mass dependence}

We determined how the discovered trends depend on galaxy stellar mass. The stellar mass affects the SFR of galaxies: more massive galaxies also form fewer stars, possibly because they have formed earlier (an effect known as 'downsizing', see e.g. Cowie et al. 1996; Bolzonella et al. 2010; Renzini 2006; Thomas et al. 2005; Pozzetti et al. 2010; Cimatti et al. 2006; Cucciati et al. 2006). Moreover, spin alignment depends on mass as well: more massive galaxies lie perpendicularly to filaments, and lower-mass galaxies are preferentially aligned, as we outlined in Sect. 3.2.2. In order to confirm whether the trends we observe are due specifically to galaxies in a given mass range (either high-mass or low-mass), we separately derived the distributions shown in Fig. 6 for high- and low-mass galaxies. This is shown in Fig. 12.

When galaxies are divided according to mass, the most striking feature is that in the case of SFR-related quantities (e.g. SFR, 

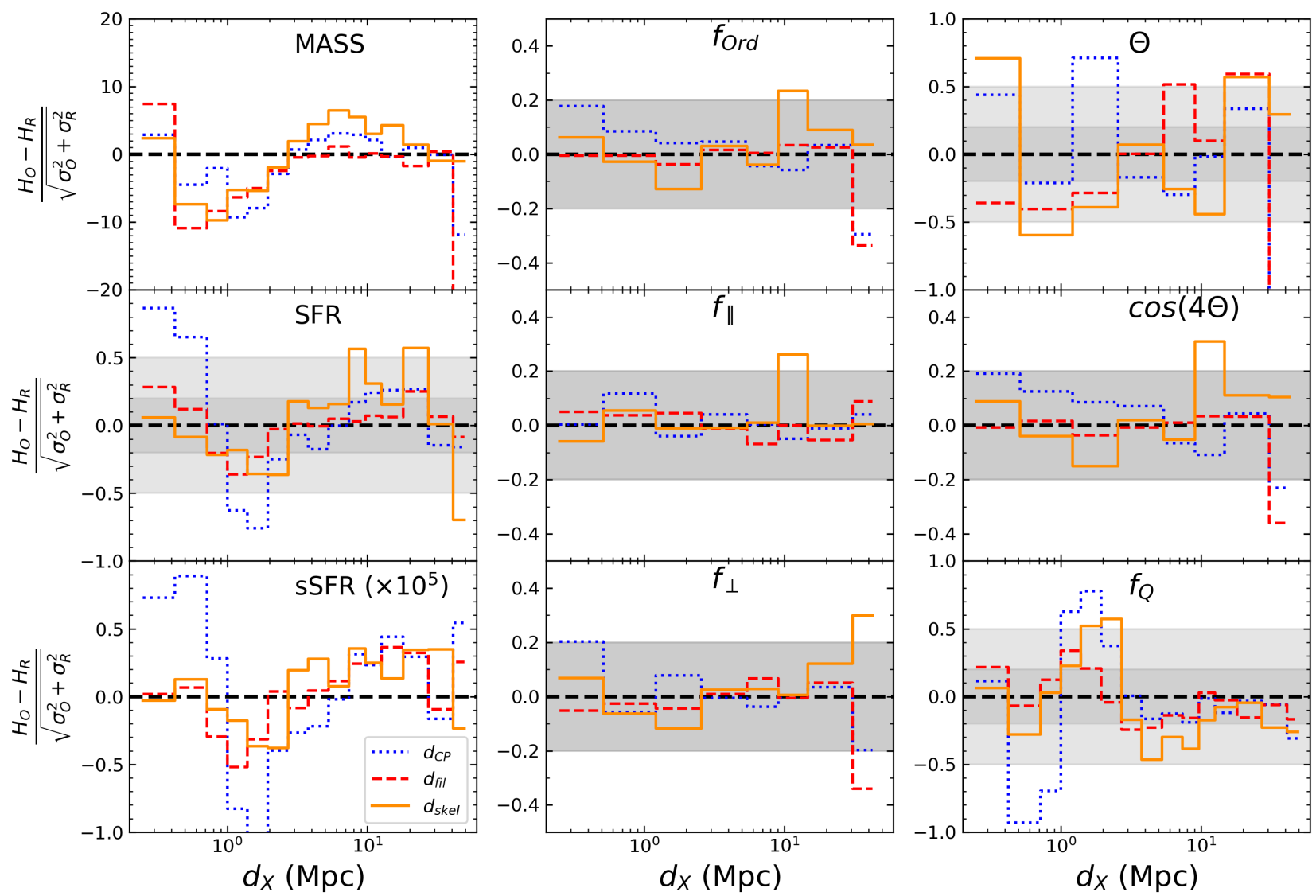

Fig. 11. Differences between the original distributions $H_{\mathrm{O}}$ and the re-shuffled ones $H_{\mathrm{R}}$, normalised to the sum in quadrature of their errors $\left(\sigma_{\mathrm{O}}\right.$ and $\sigma_{\mathrm{R}}$, respectively) for the quantities $\left\langle M^{*}\right\rangle,\langle\mathrm{SFR}\rangle,\langle\mathrm{sSFR}\rangle,\langle\theta\rangle,\langle\cos (4 \theta)\rangle$, fraction of quenched galaxies $\left(f_{\mathrm{Q}}\right)$, fraction of ordered galaxies $\left(f_{\text {Ord }}\right)$, fraction of parallel galaxies $\left(f_{\|}\right)$, and fraction of perpendicular galaxies $\left(f_{\perp}\right)$ as a function of the distances from the features of the cosmic web $d_{\text {fil }}$ (dashed red line in every panel), $d_{\mathrm{CP}}$ (dotted blue line in every panel), and $d_{\text {skel }}$ (solid orange line in every panel). In the case of $d_{\mathrm{CP}}$ and $d_{\text {skel }}$, only galaxies outside of filaments $\left(d_{\text {fil }} \geq 1 \mathrm{Mpc}\right)$ and inside filaments $\left(d_{\mathrm{fil}} \leq 1 \mathrm{Mpc}\right)$ have been considered, respectively. In all panels except for $\left\langle M^{*}\right\rangle$ and $\langle$ SSFR $\rangle$ (where they would have encompassed the whole range on the $y$-axis), light grey and dark grey areas show the $0.5 \sigma$ and $0.2 \sigma$ range, respectively.

$\mathrm{SSFR}$, and $f_{\mathrm{Q}}$ ), a trend with the distances from the structures is visible only for low-mass galaxies. High-mass galaxies generally show a lower amount of (specific-)SFR and a higher quenched fraction, but the distributions are uniform throughout the ranges of distances we explored. On the other hand, for low-mass galaxies, the same trends with distances are recovered as for the general population. This agrees with a scenario in which quenching processes can be separated into mass- and environment-driven process (e.g. Peng et al. 2010). Environmental quenching primarily affects low-mass galaxies, and mass quenching primarily affects high-mass galaxies. The star-formation activity for lowmass galaxies decreases with decreasing distance from the nodes (either following the filaments or considered isotropically) and with decreasing distance from the axis of the filaments. However, the possibility of distinguishing between structures is reduced, and the distributions with respect to $d_{\text {fil }}$ and $d_{\mathrm{CP}}$ largely overlap. In the case of mass distributions, weak trends with distances from structures are maintained for high- and low-mass galaxies, and the curves largely overlap. The only exceptions are the $\left\langle M^{*}\right\rangle$ distribution for high-mass galaxies and the $\langle\mathrm{SFR}\rangle,\langle\mathrm{sSFR}\rangle$, and $f_{\mathrm{Q}}$ distributions for low-mass galaxies as a function of $d_{\text {skel }}$, which show that massive galaxies in filaments have a higher mass and low-mass galaxies have a lower SFR than the remaining galaxy population. Spin-related quantities generally do not show differences between high- and low-mass galaxies, except for indications that the distributions of high-mass galaxies are shifted at higher values of $\theta$, with a possible faint trend for $\theta$ to decrease with $d_{\text {fil }}$ and $d_{\text {skel }}$. This is consistent with the scenario that the spin of high-mass galaxies is more perpendicular to filaments in general and especially close to structures, and it also fits within the theoretical framework laid out in Laigle et al. (2015), where gas accretion onto haloes larger than the vorticity quadrant of filaments can affect the direction of the spin. In our case, galaxies are more likely to cross several vorticity quadrants at small $d_{\text {fil }}$, while massive galaxies have had more time to accrete matter in this environment.

In Fig. 13 we show the normalised distributions of galaxy properties as a function of the distance to the structures. As several trends that were visible for the general population are absent in the case of high-mass galaxies, we only show the distributions for low-mass galaxies. In this case, trends with the distances from the LSS features are preserved, and SFRrelated quantities show the strongest variation with the distances with respect to structures. This agrees with what was found previously.

\subsection{Dividing galaxies by spin parameter}

We further refined our analysis by studying the two distinct populations of high-spin parameter and low-spin parameter galaxies, 

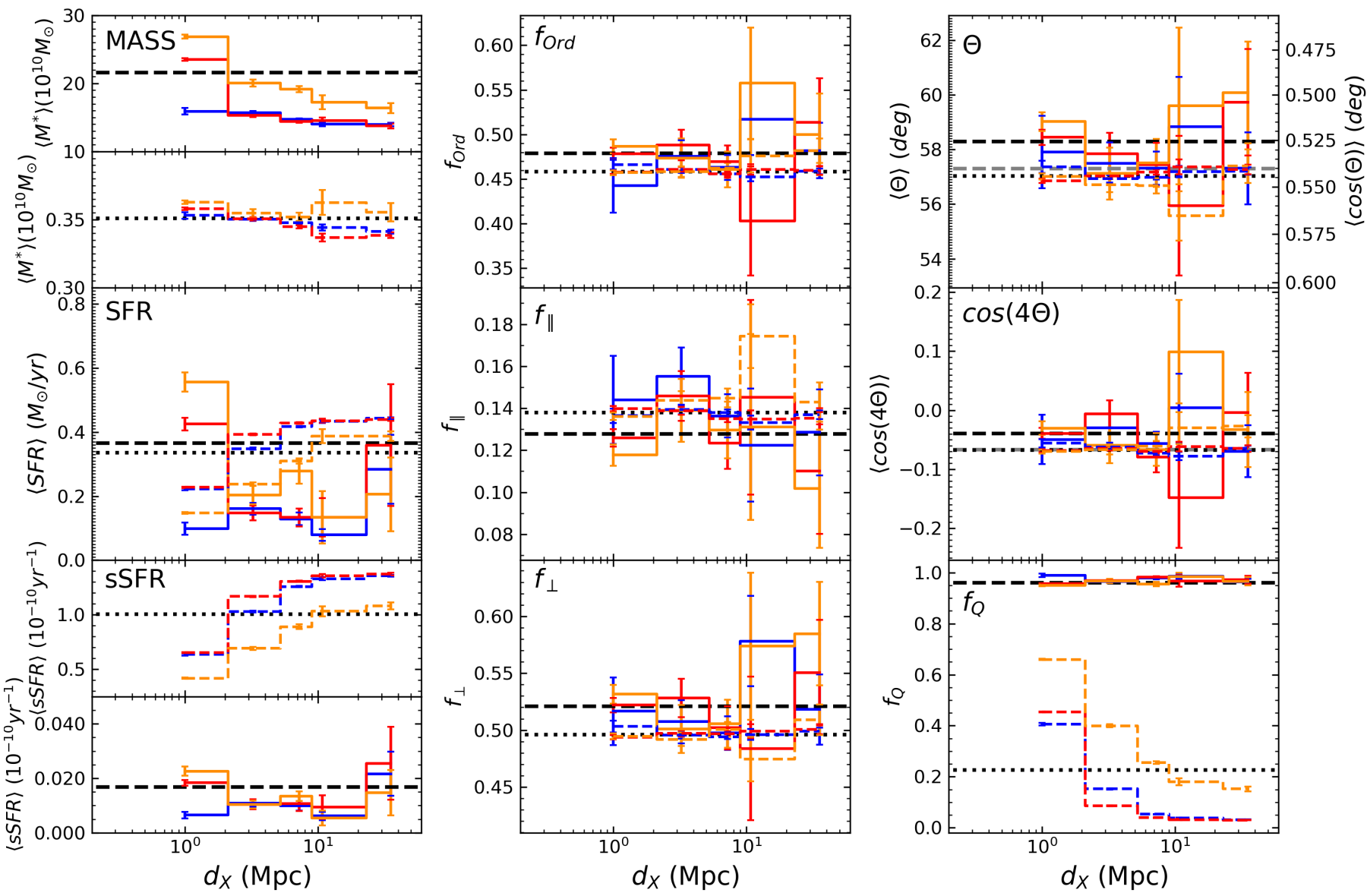

Fig. 12. Distributions of $\left\langle M^{*}\right\rangle,\langle\mathrm{SFR}\rangle,\langle\mathrm{sSFR}\rangle,\langle\theta\rangle,\langle\cos (4 \theta)\rangle$, fraction of quenched galaxies $\left(f_{\mathrm{Q}}\right)$, fraction of ordered galaxies $\left(f_{\text {Ord }}\right)$, fraction of parallel galaxies $\left(f_{\|}\right)$, and fraction of perpendicular galaxies $\left(f_{\perp}\right)$ as a function of the distances from the features of the cosmic web $d_{\text {fil }}($ red line in every panel), $d_{\mathrm{CP}}$ (blue line in every panel), and $d_{\text {skel }}$ (orange line in every panel) and split between high- and low-mass galaxies. In the case of $d_{\mathrm{CP}}$ and $d_{\text {skel }}$, only galaxies outside of filaments $\left(d_{\mathrm{fil}} \geq 1 \mathrm{Mpc}\right)$ and inside filaments $\left(d_{\mathrm{fil}} \leq 1 \mathrm{Mpc}\right)$ have been considered, respectively. Error bars on the distributions have been computed through bootstrap resampling. The dashed black (dotted) line in every panel is the average of the considered quantity in the full simulation box considering only high-mass (low-mass) galaxies. In the top and middle panel of the central column, the grey line is $\bar{\theta}$ and $\overline{\cos (4 \theta)}$, computed given Eqs. (3) and (4) for the total subhalo sample. In each panel, solid lines refer to high-mass galaxies and dashed lines to low-mass galaxies. In the case of mass and sSFR, the panel has been split in two to take the very different ranges on the $y$-axis occupied by the distributions into account. In every panel, the first distance bin we considered extends all the way to zero for all distances, but it is cut because the $x$-axis is in logarithmic scale.

divided using the percentiles of the Bullock parameter distribution and introduced in Sect. 3.2.2. Figure 4 showed that highspin parameter galaxies carry most of the signal related to spin alignment, especially when high- and low-mass galaxies are considered separately. In the following, we therefore focus only on this sub-sample.

In this case, the situation is similar to what we obtained in Fig. 12 for the total galaxy population. Trends with the distances from structures are still visible for $M^{*}$ and SFR-related quantities, but the large error bars and a large overlap of the distributions of spin-related quantities prevent us from detecting any secure trend.

Figure 14 shows the normalised distributions of galaxy properties for low-mass galaxies as in Fig. 13 for the case when only high-spin parameter galaxies are considered. Trends with the distances from the structures are again visible for low-mass highspin parameter galaxies. The SFR-related quantity $f_{\mathrm{Q}}$ indeed shows the largest variation with respect to the distances from the structures. In the case of $d_{\text {skel }}$ and $d_{\text {fil }}$, this is also matched by $\langle\cos (4 \theta)\rangle$.

\section{Concluding remarks}

We used the IllustrisTNG simulation, coupled with the DisPerSE cosmic web extractor, to analyse the effect of different features of the cosmic web (clusters and filaments) on galaxy properties (mass, star formation, and the direction of the angular momentum vector). Although the relation of these properties to the features of the cosmic web has been independently analysed in great detail in the literature for the three quantities, this is the first time that they are compared and contrasted in an extensive and comprehensive way. In particular, we explored the possibility that one or more galaxy properties show different trends with the various cosmic web features and present a science case for their use to improve the detection of the cosmic web. Based on our analysis, we are able to draw the following conclusions:

1. When the distributions of galaxy properties are considered separately, SFR-related quantities allow us to distinguish between $d_{\text {fil }}$ (proxy for the accretion onto filaments) and $d_{\mathrm{CP}}$ and $d_{\text {skel }}$, proxies for the accretion onto nodes (further separated between the isotropic case and flowing inside the 
Low-mass

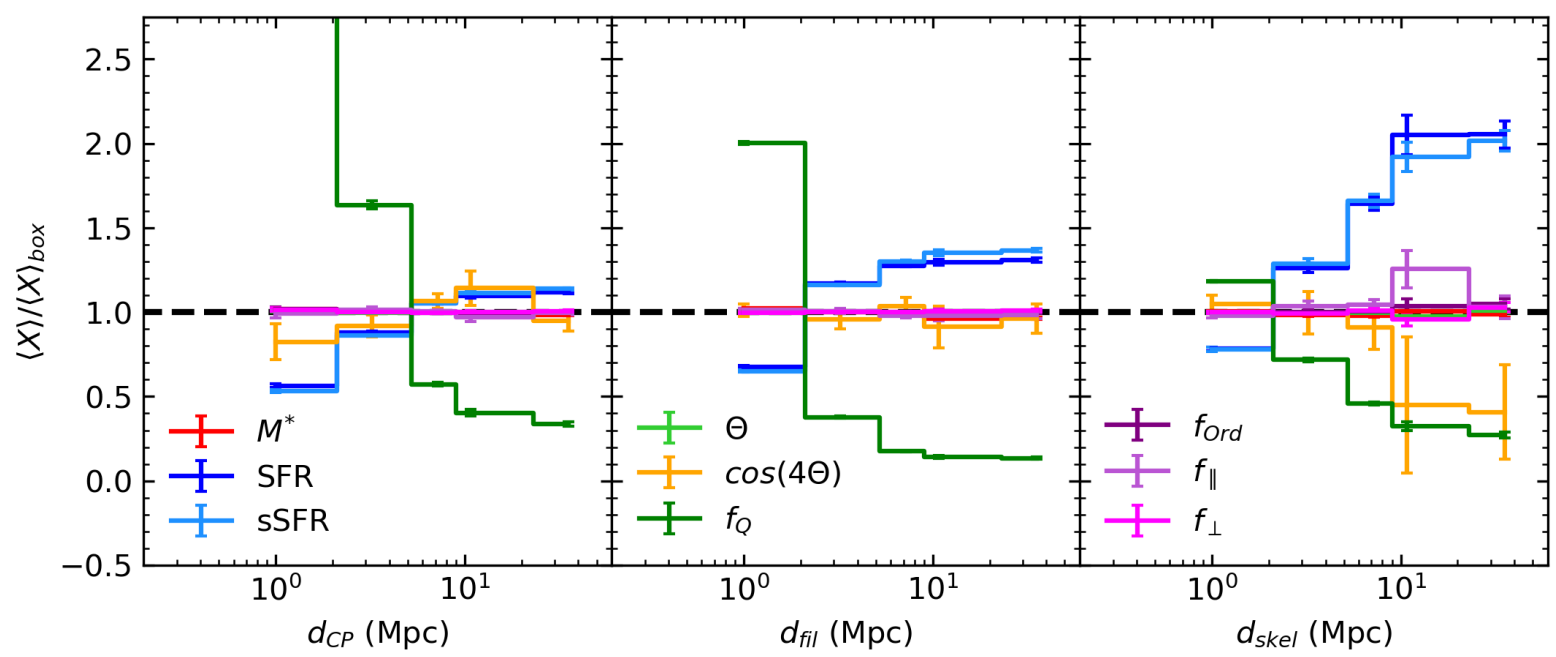

Fig. 13. Normalised distributions (expressed as $\frac{\langle X\rangle}{\langle X\rangle_{\text {box }}}$ ) with respect to distances $d_{\mathrm{CP}}$ (left panel), $d_{\mathrm{fil}}$ (middle panel), and $d_{\mathrm{skel}}$ (right panel). The quantities considered in each panel $(\langle X\rangle)$ are $\left\langle M^{*}\right\rangle$ (red), $\langle\mathrm{SFR}\rangle$ (dark blue), $\langle\mathrm{sSFR}\rangle$ (light blue), $\langle\theta\rangle$ (light green), $\langle\cos (4 \theta)\rangle$ (orange), $f_{\mathrm{Q}}(\mathrm{dark}$ green), $f_{\text {Ord }}$ (dark purple), $f_{\|}$(light purple), and $f_{\perp}$ (magenta). $\langle X\rangle_{\text {box }}$ indicates the average of the quantity taken including all low-mass subhaloes in the box (for $d_{\text {fil }}$ ), only those outside filaments $\left(d_{\mathrm{CP}}\right)$, and only those inside filaments $\left(d_{\text {skel }}\right)$ over the full simulation volume. This figure only refers to low-mass galaxies. Error bars on the distributions have been computed through bootstrap resampling. In every panel, the first distance bin considered extends all the way to zero for all distances, but it is cut because the $x$-axis is in logarithmic scale.

\section{Low-mass, high-spin parameter}

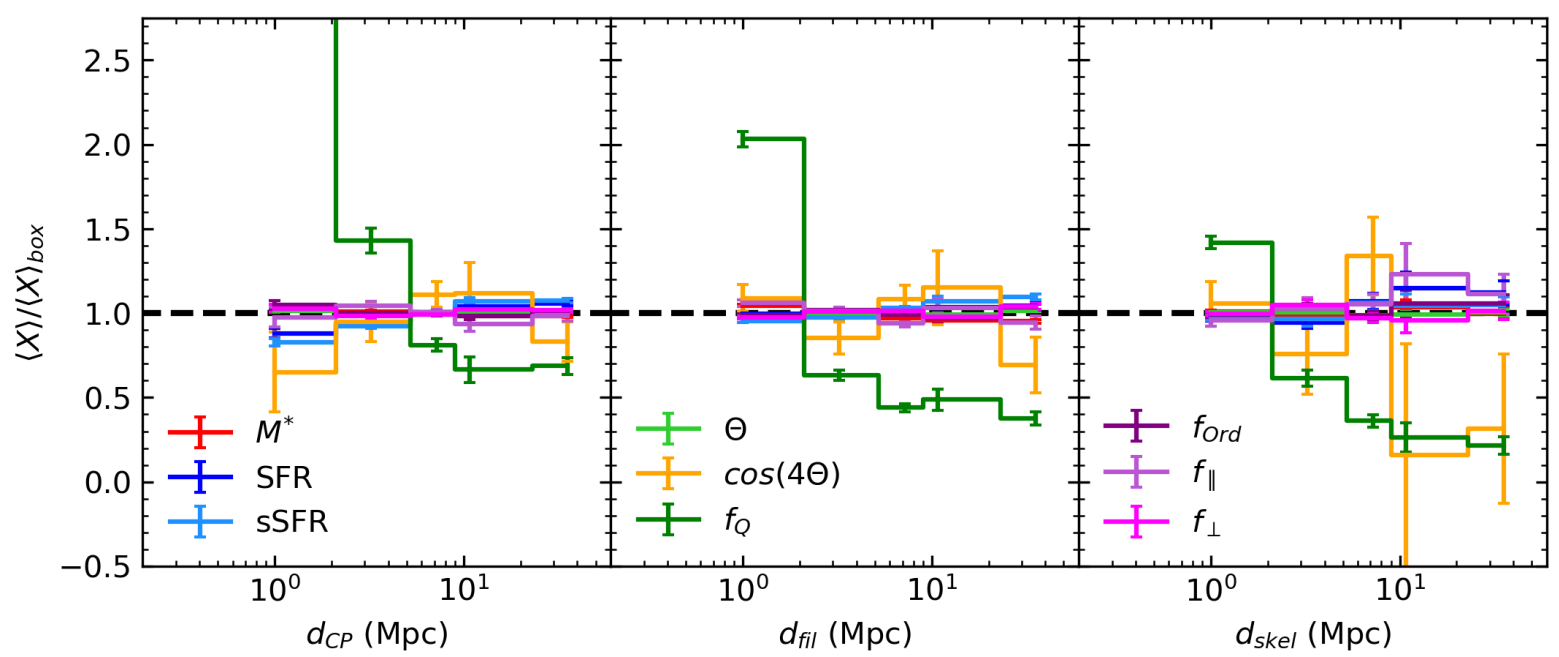

Fig. 14. Normalised distributions (expressed as $\frac{\langle X\rangle}{\langle X\rangle_{\mathrm{bov}}}$ ) with respect to distances $d_{\mathrm{CP}}$ (left panel), $d_{\mathrm{fil}}$ (middle panel), and $d_{\mathrm{skel}}$ (right panel). The quantities considered in each panel $(\langle X\rangle)$ are $\left\langle M^{*}\right\rangle$ (red), $\langle\mathrm{SFR}\rangle$ (dark blue), $\langle\mathrm{sSFR}\rangle$ (light blue), $\langle\theta\rangle$ (light green), $\langle\cos (4 \theta)\rangle$ (orange), $f_{\mathrm{Q}}(\mathrm{dark}$ green), $f_{\text {Ord }}$ (dark purple), $f_{\|}$(light purple), and $f_{\perp}$ (magenta). $\langle X\rangle_{\text {box }}$ indicates the average of the quantity taken including all low-mass, high-spin parameter subhaloes in the box (for $\left.d_{\text {fil }}\right)$, only those outside filaments $\left(d_{\mathrm{CP}}\right)$, and only those inside filaments $\left(d_{\text {skel }}\right)$ over the full simulation volume. Only low-mass high-spin parameter galaxies are considered in this figure. In every panel, the first distance bin considered extends all the way to zero for all distances, but it is cut because the $x$-axis is in logarithmic scale.

filaments). Mass- and spin-related quantities seem to allow only for a distinction between $d_{\mathrm{CP}}$ and $d_{\text {fil }}$ with respect to $d_{\text {skel. }}$.

2. When the three distances are considered separately, the distributions of SFR-related quantites show the largest variation with respect to each distance. This is further confirmation that they are the best tracers for $d_{\mathrm{CP}}, d_{\mathrm{fil}}$, and $d_{\text {skel }}$. Mass shows a smaller variation, which confirms it as the second choice for a tracer quantity, and spin-related quantities show the lowest amount of variation and are therefore the weakest tracer.
3. SFR-related quantities also show a strong dependence on the local environment of galaxies, which may prevent their use as tracers to improve the detection of the cosmic web. On the other hand, spin-related quantities are more robust with respect to the effect of local density. Although the strength of the signal of the recovered trends is lower, their use could provide a detection of the cosmic web in a way that is more independent of the local density.

4. When galaxies are separated by mass, the trends explored in the general case are visible mainly for the low-mass galaxy 
population. However, in this case as well, SFR-related quantities allow for the best separation between the distances to the various structures.

5. If only high-spin parameter subhaloes are selected and further separated in mass, trends in the distribution of quantities with the considered distances are more difficult to detect. They seem to be visible only in the case of $d_{\mathrm{CP}}$ and $d_{\mathrm{skel}}$, where $f_{\mathrm{Q}}$ and $\cos (4 \theta)$ trace structures equally well.

With a renewed interest in the study of the cosmic web and several large-scale upcoming galaxy surveys underway, it is vital to improve our understanding of how the cosmic web affects galaxy evolution and how we can better use galaxy properties to detect the cosmic web. The results reported here will greatly benefit from larger samples of subhaloes obtained from future larger simulative efforts, which will allow increasing the statistical significance of the trends that are most difficult to detect (e.g. those related to the direction of the angular momentum of galaxies). In addition, further analyses targeting these same trends for particular sub-samples of simulated galaxies (e.g. in certain magnitude ranges or separated by morphological type) that reproduce observed data sets will ensure that this context may be applied to future expected surveys. We also aim to perform analyses similar to this work while also better characterising the various structures of the cosmic web, for instance, differentiating between thick and thin filaments. Our final goal is to apply this framework to real data sets in the future.

Acknowledgements. We would like to thank the anonymous referee for the careful comments, which improved the quality of the paper. NM would like to thank Thibaud Moutard, Louis Legrand, Tony Bonnaire, and Alexander Kolodzig for useful and fruitful discussions during the performing of the analysis exposed in this work. This research has been supported by the funding for the ByoPiC project from the European Research Council (ERC) under the European Union's Horizon 2020 research and innovation programme grant agreement ERC-2015-AdG 695561. We would like to thank the IllustrisTNG team for publicly releasing the full simulated snapshots and halo catalogues. NM would like to thank Thierry Sousbie and Christophe Pichon for developing and making freely available the DisPerSE code.

\section{References}

Abazajian, K. N., Adelman-McCarthy, J. K., Agüeros, M. A., et al. 2009, ApJS, 182,543

Alam, S., Albareti, F. D., Allende Prieto, C., et al. 2015, ApJS, 219, 12

Alpaslan, M., Grootes, M., Marcum, P. M., et al. 2016, MNRAS, 457, 2287

Aragon Calvo, M. A., Neyrinck, M. C., \& Silk, J. 2019, Open J. Astrophys., 2, 7

Aragón-Calvo, M. A., van de Weygaert, R., Jones, B. J. T., \& van der Hulst, J. M. 2007, ApJ, 655, L5

Aragón-Calvo, M. A., van de Weygaert, R., \& Jones, B. J. T. 2010, MNRAS, 408, 2163

Arnaud, M., Pratt, G. W., Piffaretti, R., et al. 2010, A\&A, 517, A92

Baugh, C. M., Gonzalez-Perez, V., Lagos, C. D. P., et al. 2019, MNRAS, 483, 4922

Bett, P. E., \& Frenk, C. S. 2012, MNRAS, 420, 3324

Bilicki, M., Peacock, J. A., Jarrett, T. H., et al. 2016, ApJS, 225, 5

Blanton, M. R., \& Moustakas, J. 2009, ARA\&A, 47, 159

Bolzonella, M., Kovač, K., Pozzetti, L., et al. 2010, A\&A, 524, A76

Bond, J. R., Kofman, L., \& Pogosyan, D. 1996, Nature, 380, 603

Bonjean, V., Aghanim, N., Douspis, M., Malavasi, N., \& Tanimura, H. 2020, A\&A, 638, A75

Boselli, A., \& Gavazzi, G. 2006, PASP, 118, 517

Boselli, A., \& Gavazzi, G. 2014, A\&ARv, 22, 74

Bryant, J. J., Owers, M. S., Robotham, A. S. G., et al. 2015, MNRAS, 447, 2857

Bullock, J. S., Dekel, A., Kolatt, T. S., et al. 2001, ApJ, 555, 240

Bundy, K., Bershady, M. A., Law, D. R., et al. 2015, ApJ, 798, 7

Catelan, P., \& Theuns, T. 1996, MNRAS, 282, 436

Chen, Y.-C., Ho, S., Tenneti, A., et al. 2015, MNRAS, 454, 3341

Chen, Y.-C., Ho, S., Mandelbaum, R., et al. 2017, MNRAS, 466, 1880

Chen, Y.-C., Ho, S., Blazek, J., et al. 2019, MNRAS, 485, 2492

Cimatti, A., Daddi, E., \& Renzini, A. 2006, A\&A, 453, L29
Codis, S., Pichon, C., Devriendt, J., et al. 2012, MNRAS, 427, 3320 Codis, S., Pichon, C., \& Pogosyan, D. 2015, MNRAS, 452, 3369

Codis, S., Jindal, A., Chisari, N. E., et al. 2018, MNRAS, 481, 4753

Cowie, L. L., Songaila, A., Hu, E. M., \& Cohen, J. G. 1996, AJ, 112, 839

Crittenden, R. G., Natarajan, P., Pen, U.-L., \& Theuns, T. 2001, ApJ, 559, 552

Croom, S. M., Lawrence, J. S., Bland-Hawthorn, J., et al. 2012, MNRAS, 421, 872

Cucciati, O., Iovino, A., Marinoni, C., et al. 2006, A\&A, 458, 39

Davé, R., Anglés-Alcázar, D., Narayanan, D., et al. 2019, MNRAS, 486, 2827

Davidzon, I., Cucciati, O., Bolzonella, M., et al. 2016, A\&A, 586, A23

de Lapparent, V., Geller, M. J., \& Huchra, J. P. 1986, ApJ, 302, L1

De Lucia, G. 2007, in Cosmic Frontiers, eds. N. Metcalfe, \& T. Shanks, ASP Conf. Ser., 379, 257

Dekel, A., Porciani, C., Kolatt, T. S., et al. 2001, in Galaxy Disks and Disk Galaxies, eds. J. G. Funes, \& E. M. Corsini, ASP Conf. Ser., 230, 565

Dolag, K., Borgani, S., Murante, G., \& Springel, V. 2009, MNRAS, 399, 497

Donnari, M., Pillepich, A., Nelson, D., et al. 2021, MNRAS, 506, 4760

Doroshkevich, A. G. 1970, Astrophysics, 6, 320

Dressler, A. 1980, ApJ, 236, 351

Dressler, A. 1986, ApJ, 301, 35

Driver, S. P., Norberg, P., Baldry, I. K., et al. 2009, Astron. Geophys., 50, 5.12

Dubois, Y., Pichon, C., Welker, C., et al. 2014, MNRAS, 444, 1453

Dutton, A. A., \& van den Bosch, F. C. 2012, MNRAS, 421, 608

Galárraga-Espinosa, D., Aghanim, N., Langer, M., Gouin, C., \& Malavasi, N. 2020, A\&A, 641, A173

Ganeshaiah Veena, P., Cautun, M., van de Weygaert, R., et al. 2018, MNRAS, 481,414

Ganeshaiah Veena, P., Cautun, M., Tempel, E., van de Weygaert, R., \& Frenk, C. S. 2019, MNRAS, 487, 1607

Ganeshaiah Veena, P., Cautun, M., van de Weygaert, R., Tempel, E., \& Frenk, C. S. 2021, MNRAS, 503, 2280

Gouin, C., Aghanim, N., Bonjean, V., \& Douspis, M. 2020, A\&A, 635, A195

Hahn, O., Carollo, C. M., Porciani, C., \& Dekel, A. 2007a, MNRAS, 381, 41

Hahn, O., Porciani, C., Carollo, C. M., \& Dekel, A. 2007b, MNRAS, 375, 489

Hahn, O., Teyssier, R., \& Carollo, C. M. 2010, MNRAS, 405, 274

Hellwing, W. A., Cautun, M., van de Weygaert, R., \& Jones, B. T. 2021, Phys. Rev. D, 103, 063517

Hernquist, L. 1990, ApJ, 356, 359

Huchra, J. P., Macri, L. M., Masters, K. L., et al. 2012, ApJS, 199, 26

Ilbert, O., McCracken, H. J., Le Fèvre, O., et al. 2013, A\&A, 556, A55

Khandai, N., Di Matteo, T., Croft, R., et al. 2015, MNRAS, 450, 1349

Kraljic, K., Arnouts, S., Pichon, C., et al. 2018, MNRAS, 474, 547

Kraljic, K., Pichon, C., Dubois, Y., et al. 2019, MNRAS, 483, 3227

Kraljic, K., Davé, R., \& Pichon, C. 2020, MNRAS, 493, 362

Krolewski, A., Ho, S., Chen, Y.-C., et al. 2019, ApJ, 876, 52

Kuutma, T., Tamm, A., \& Tempel, E. 2017, A\&A, 600, L6

Laigle, C., Pichon, C., Codis, S., et al. 2015, MNRAS, 446, 2744

Laigle, C., McCracken, H. J., Ilbert, O., et al. 2016, ApJS, 224, 24

Laigle, C., Pichon, C., Arnouts, S., et al. 2018, MNRAS, 474, 5437

Malavasi, N., Arnouts, S., Vibert, D., et al. 2017a, MNRAS, 465, 3817

Malavasi, N., Pozzetti, L., Cucciati, O., et al. 2017b, MNRAS, 470, 1274

Malavasi, N., Aghanim, N., Tanimura, H., Bonjean, V., \& Douspis, M. 2020a, A\&A, 634, A30

Malavasi, N., Aghanim, N., Douspis, M., Tanimura, H., \& Bonjean, V. 2020b, A\&A, 642, A19

Marinacci, F., Vogelsberger, M., Pakmor, R., et al. 2018, MNRAS, 480, 5113

McCullagh, N., Norberg, P., Cole, S., et al. 2017, ArXiv e-prints [arXiv:1705.01988]

Moutard, T., Arnouts, S., Ilbert, O., et al. 2016a, A\&A, 590, A103

Moutard, T., Arnouts, S., Ilbert, O., et al. 2016b, A\&A, 590, A102

Moutard, T., Sawicki, M., Arnouts, S., et al. 2018, MNRAS, 479, 2147

Moutard, T., Malavasi, N., Sawicki, M., Arnouts, S., \& Tripathi, S. 2020, MNRAS, 495, 4237

Nagai, D., Kravtsov, A. V., \& Vikhlinin, A. 2007, ApJ, 668, 1

Naiman, J. P., Pillepich, A., Springel, V., et al. 2018, MNRAS, 477, 1206

Navarro, J. F., Frenk, C. S., \& White, S. D. M. 1997, ApJ, 490, 493

Nelson, D., Pillepich, A., Genel, S., et al. 2015, Astron. Comput., 13, 12

Nelson, D., Pillepich, A., Springel, V., et al. 2018, MNRAS, 475, 624

Nelson, D., Springel, V., Pillepich, A., et al. 2019, Comput. Astrophys. Cosmol., 6,2

Pahwa, I., Libeskind, N. I., Tempel, E., et al. 2016, MNRAS, 457, 695

Pandya, V., Primack, J., Behroozi, P., et al. 2019, MNRAS, 488, 5580

Paz, D. J., Stasyszyn, F., \& Padilla, N. D. 2008, MNRAS, 389, 1127

Peebles, P. J. E. 1969, ApJ, 155, 393

Peng, Y.-J., Lilly, S. J., Kovač, K., et al. 2010, ApJ, 721, 193

Pillepich, A., Nelson, D., Hernquist, L., et al. 2018, MNRAS, 475, 648

Planck Collaboration XIII. 2016, A\&A, 594, A13

Porciani, C., Dekel, A., \& Hoffman, Y. 2002, MNRAS, 332, 325 
Pozzetti, L., Bolzonella, M., Zucca, E., et al. 2010, A\&A, 523, A13

Renzini, A. 2006, ARA\&A, 44, 141

Rong, Y., Liu, Y., \& Zhang, S.-N. 2016, MNRAS, 455, 2267

Rost, A., Stasyszyn, F., Pereyra, L., \& Martínez, H. J. 2020, MNRAS, 493, 1936

Rykoff, E. S., Rozo, E., Busha, M. T., et al. 2014, ApJ, 785, 104

Salerno, J. M., Martínez, H. J., Muriel, H., et al. 2020, MNRAS, 493, 4950

Schaap, W. E., \& van de Weygaert, R. 2000, A\&A, 363, L29

Scodeggio, M., Guzzo, L., Garilli, B., et al. 2018, A\&A, 609, A84

Song, H., Laigle, C., Hwang, H. S., et al. 2021, MNRAS, 501, 4635

Sousbie, T. 2011, MNRAS, 414, 350

Sousbie, T., Pichon, C., \& Kawahara, H. 2011, MNRAS, 414, 384

Springel, V. 2010, MNRAS, 401, 791

Springel, V., White, S. D. M., Tormen, G., \& Kauffmann, G. 2001, MNRAS, 328,726

Springel, V., Pakmor, R., Pillepich, A., et al. 2018, MNRAS, 475, 676

Strateva, I., Ivezić, Ž., Knapp, G. R., et al. 2001, AJ, 122, 1861

Tempel, E., \& Libeskind, N. I. 2013, ApJ, 775, L42

Tempel, E., Stoica, R. S., \& Saar, E. 2013, MNRAS, 428, 1827

Teyssier, R., Pires, S., Prunet, S., et al. 2009, A\&A, 497, 335
Thomas, D., Maraston, C., Bender, R., \& Mendes de Oliveira, C. 2005, ApJ, 621, 673

Trowland, H. E., Lewis, G. F., \& Bland-Hawthorn, J. 2013, ApJ, 762, 72

Trujillo, I., Carretero, C., \& Patiri, S. G. 2006, ApJ, 640, L111

van de Weygaert, R., \& Schaap, W. 2009, in The Cosmic Web: Geometric Analysis, eds. V. J. Martínez, E. Saar, E. Martínez-González, \& M. J. PonsBordería, 665, 291

Vogelsberger, M., Genel, S., Springel, V., et al. 2014, Nature, 509, 177

Wang, P., \& Kang, X. 2017, MNRAS, 468, L123

Wang, P., Libeskind, N. I., Tempel, E., et al. 2020, ApJ, 900, 129

Welker, C., Devriendt, J., Dubois, Y., Pichon, C., \& Peirani, S. 2014, MNRAS, 445, L46

Welker, C., Bland-Hawthorn, J., Van de Sande, J., et al. 2020, MNRAS, 491, 2864

White, S. D. M. 1984, ApJ, 286, 38

York, D. G., Adelman, J., Anderson, J. E., Jr., et al. 2000, AJ, 120, 1579

Zel'dovich, Y. B. 1970a, Astrophysics, 6, 164

Zel'dovich, Y. B. 1970b, A\&A, 500, 13

Zhang, Y., Yang, X., Wang, H., et al. 2013, ApJ, 779, 160 


\section{Appendix A: Thresholding the critical point density}

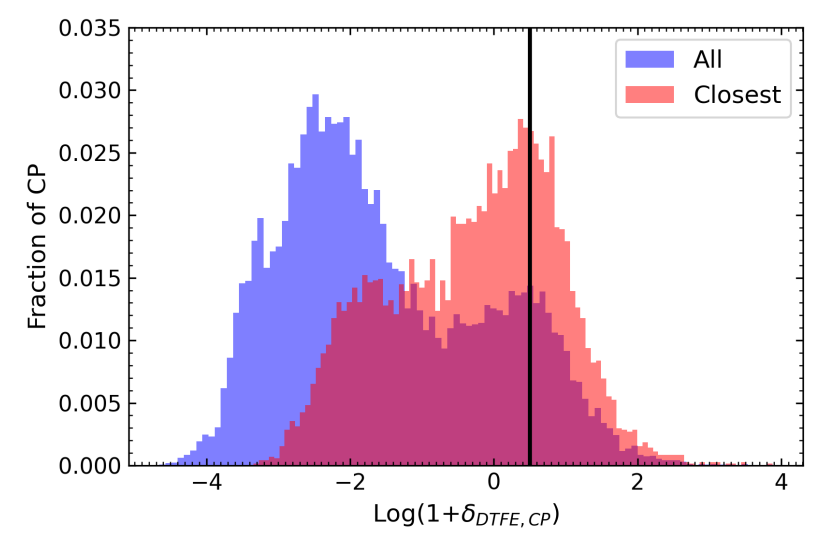

Fig. A.1. Density contrast distribution for all the critical points in the simulation box (blue) and those identified as closest to at least a subhalo (only maxima and bifurcations considered, in red) when performing the measurement of $d_{\mathrm{CP}}$. The vertical black line shows the threshold in critical point density contrast we adopted.

Ins our analysis we made use of the critical points identified by DisPerSE (bifurcations and maxima) as a proxy for galaxy overdensities (groups and clusters). However, the identification and matching of these points with confirmed galaxy clusters is difficult because the distribution of these points depends to some extent on the smoothing and persistence threshold that is adopted (see e.g. Malavasi et al. 2020b). For this reason, it may be misleading to consider all maxima and bifurcations as potential clusters or groups. In order to determine how much this affects our analysis, we thresholded the critical points according to their density.
Figure A. 1 shows the distribution of $\log \left(1+\delta_{\mathrm{DTFE}, \mathrm{CP}}\right)$ for the critical points, where $\delta_{\mathrm{DTFE}, \mathrm{CP}}=\frac{\left.\rho_{\mathrm{DTFE}, \mathrm{CP}}-\rho_{\mathrm{DTFE}, \mathrm{Gal}}\right\rangle}{\left\langle\rho_{\mathrm{DTFE}, \mathrm{Gal}}\right\rangle}$. In this formula, $\rho_{\text {DTFE,CP }}$ is the value of the density computed from the Delaunay tessellation at the position of critical points, while $\rho_{\mathrm{DTFE}, \mathrm{Gal}}$ is the density computed at the position of the subhaloes. To measure the average density $\left\langle\rho_{\text {DTFE,Gal }}\right\rangle$, we used all the subhaloes in the box. Figure A.1 shows the density contrast distribution for all the critical points in the simulation box and for those associated with subhaloes, that is, the points that are found to be the closest critical point to at least one subhalo when $d_{\mathrm{CP}}$ is measured.

The density contrast distribution for all critical points is bimodal. This highlights lower-density minima and saddles and higher-density bifurcations and maxima. When critical points are attached to subhaloes, the density contrast distribution is restricted to the high-density tail because we only considered maxima and bifurcations.

We adopted a density contrast threshold of $\log (1+$ $\left.\delta_{\mathrm{DTFE}, \mathrm{CP}}\right)=0.5$ and eliminated all galaxies whose closest critical point (considering $d_{\mathrm{CP}}$ ) was below the threshold from our sample. This left a final sample of 102064 subhaloes associated with dense critical points. We re-derived the distributions shown in Figure 6 using only this subset of galaxies. The new distributions are shown in Figure A.2. In addition to the increased noise in the distributions, no major difference from the general case can be seen when only dense critical points close to subhaloes are considered. The only exception is represented by the innermost bin in the $d_{\mathrm{CP}}$ distribution of spin-related quantities, which now show a much larger fraction of parallel galaxies. However, given for example the large error bars in the distribution of $f_{\|}$ and the very short distance from the critical points we considered, this might be due to the reduced number counts in the first bin. 
High-density critical points
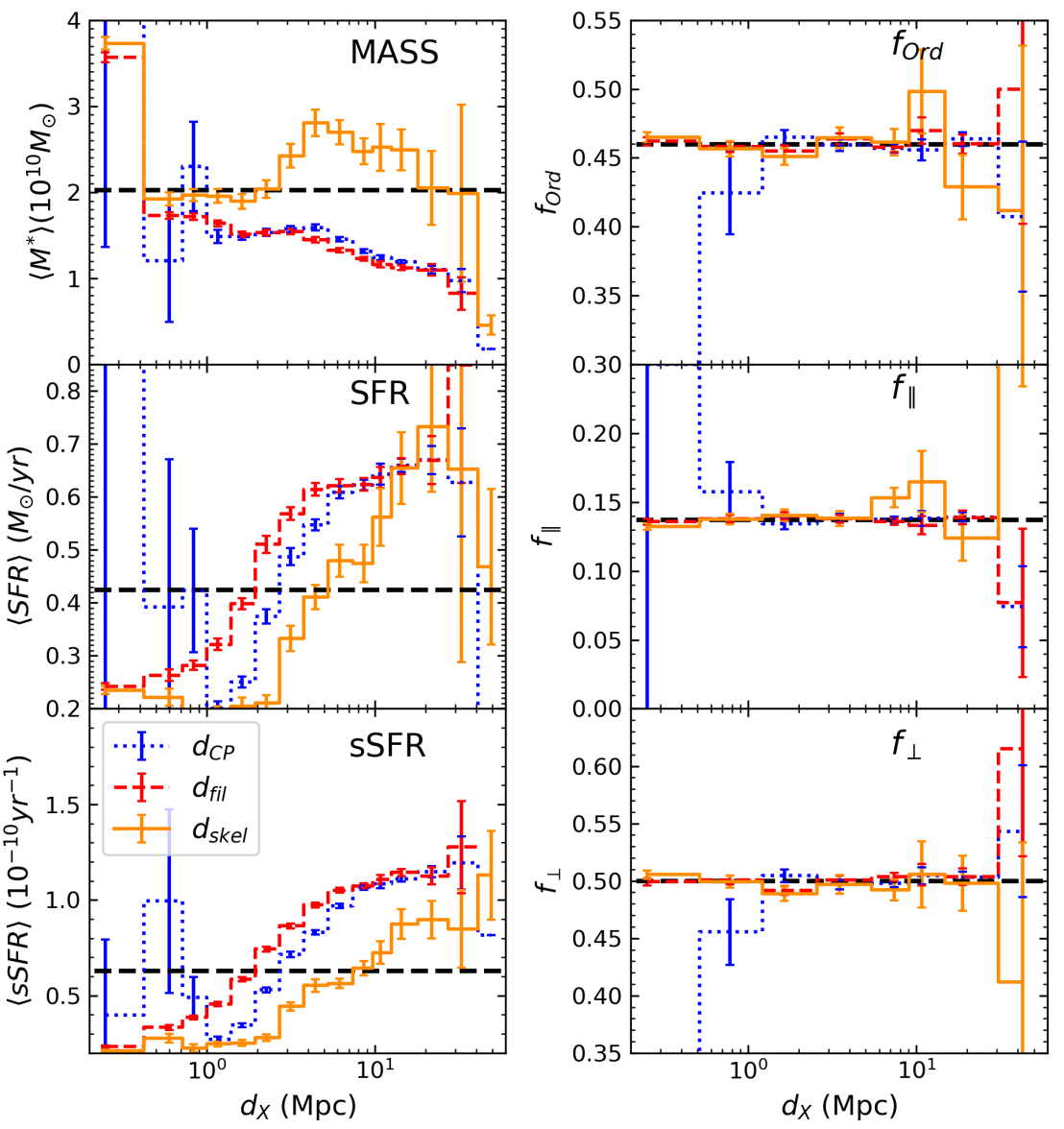

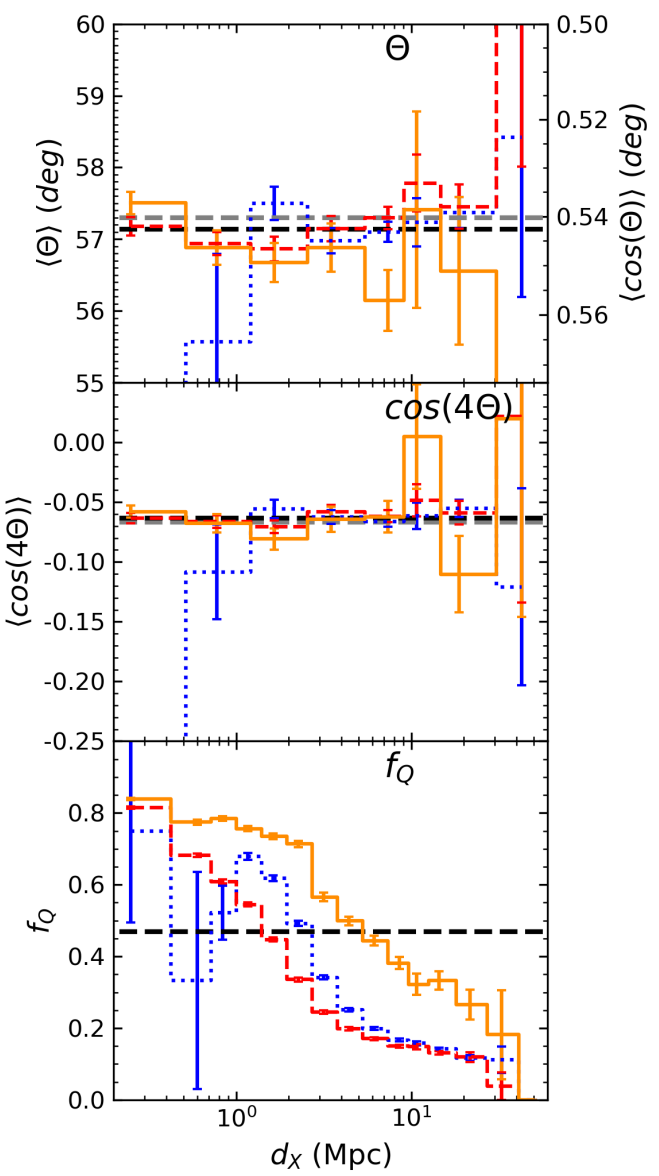

Fig. A.2. Distributions of $\left\langle M^{*}\right\rangle,\langle\mathrm{SFR}\rangle,\langle\mathrm{sSFR}\rangle,\langle\theta\rangle,\langle\cos (4 \theta)\rangle$, fraction of quenched galaxies $\left(f_{\mathrm{Q}}\right)$, fraction of ordered galaxies $\left(f_{\text {Ord }}\right)$, fraction of parallel galaxies $\left(f_{\|}\right)$, and fraction of perpendicular galaxies $\left(f_{\perp}\right)$ as a function of the distances from the features of the cosmic web $d_{\mathrm{fil}}(\mathrm{dashed}$ red line in every panel), $d_{\mathrm{CP}}$ (dotted blue line in every panel), and $d_{\text {skel }}$ (solid orange line in every panel). In the case of $d_{\mathrm{CP}}$ and $d_{\text {skel }}$, only galaxies outside of filaments $\left(d_{\mathrm{fil}} \geq 1 \mathrm{Mpc}\right)$ and inside filaments $\left(d_{\mathrm{fil}} \leq 1 \mathrm{Mpc}\right)$ have been considered, respectively. Error bars on the distributions have been computed through bootstrap resampling. The dashed black line in every panel is the average of the considered quantity in the full simulation box. In the top and middle panel of the central column, the grey line is $\bar{\theta}$ and $\overline{\cos (4 \theta)}$, computed given equations (3) and (4) for the total subhalo sample. Only subhaloes whose closer critical point (in terms of $\left.d_{\mathrm{CP}}\right)$ is above the density contrast threshold of $\log \left(1+\delta_{\mathrm{DTFE}, \mathrm{CP}}\right)=0.5$ have been considered. In every panel, the first distance bin considered extends all the way to zero for all distances, but it is cut because the $x$-axis is in logarithmic scale. In the $f_{\|}$and $f_{\perp}$ cases, the y-axes of the plots cover very different ranges. 
Appendix B: Considering different components to measure the spin

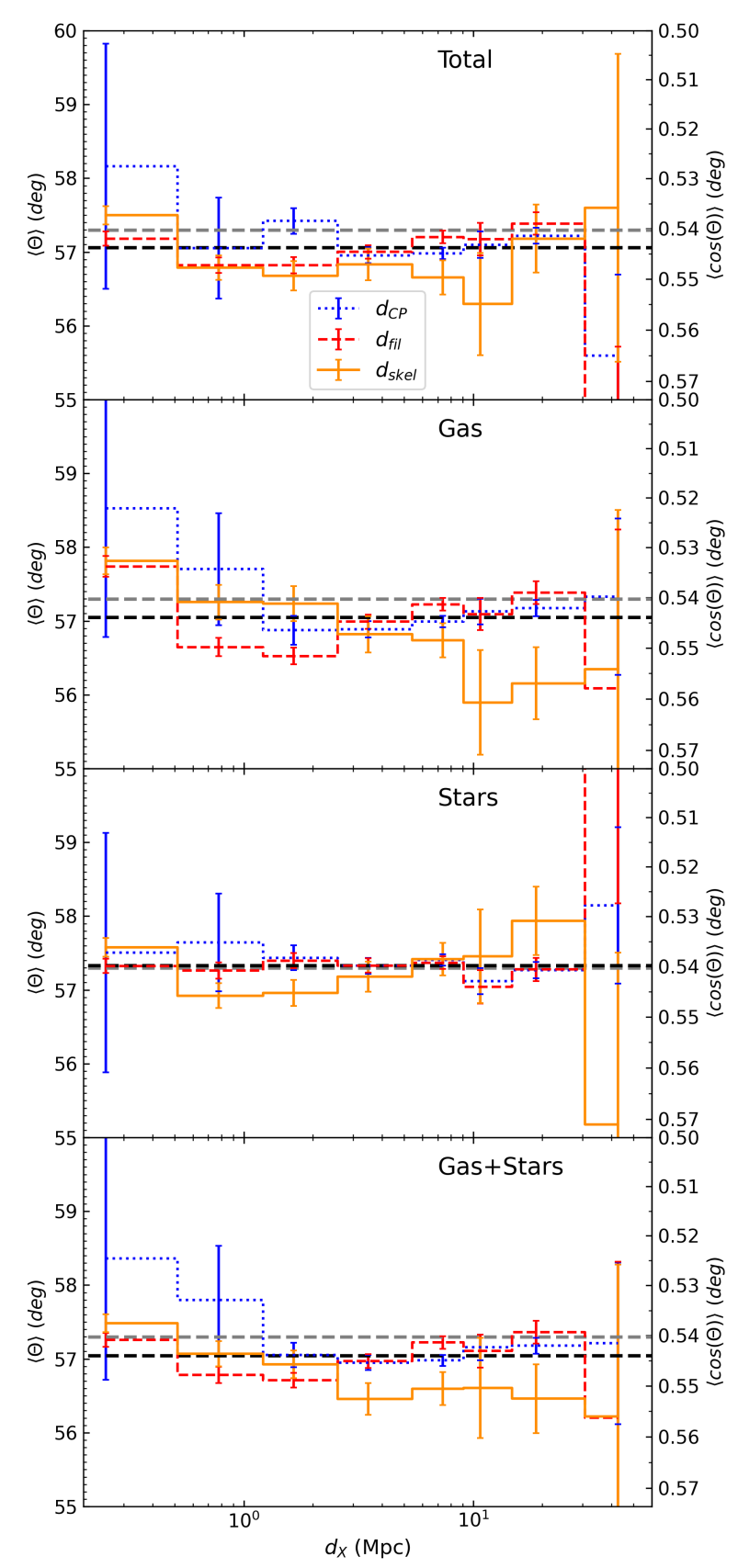

Fig. B.1. Distributions of $\langle\theta\rangle$ as a function of the distances from the features of the cosmic web $d_{\text {fil }}$ (dashed red line in every panel), $d_{\mathrm{CP}}$ (dotted blue line in every panel), and $d_{\text {skel }}$ (solid orange line in every panel). The first panel shows the distributions obtained when all subhalo components are considered (same as the top middle panel of Figure 6), the subsequent panels refer to the distributions obtained when only the gas component is considered (second panel), only the stellar component (third panel), and when both gas and stars (but not dark matter) are considered (bottom panel). The dashed black line in every panel is the average of the considered quantity in the full simulation box. In the top middle panel, the grey line is $\bar{\theta}$, computed given equation (3) for the total subhalo sample. In every panel, the first distance bin considered extends all the way to zero for all distances, but it is cut because the $x$-axis is in logarithmic scale.
As observations are generally able to only probe the baryonic components of galaxies (gas and stars) rather than their dark matter haloes, we explored the change in the trends with distances from structures for spin-related quantities when the spin is measured using only the gaseous or stellar components of subhaloes. Figure B. 1 shows the distributions of $\langle\theta\rangle$ as a function of $d_{\mathrm{CP}}, d_{\text {skel }}$, and $d_{\text {fil }}$, when the spin is measured using all the components (this is the same as the top middle panel in Figure 6), only the gas, only the stars, or both the gas and stars within each subhalo.

This figure shows that the trend that is visible in the distributions where the spin of galaxies switches from parallel to perpendicular to the filaments as galaxies flow inside filaments to reach nodes (i.e. where $\langle\theta\rangle$ increases with decreasing $d_{\text {skel }}$ ) is essentially due to the gaseous component of galaxies. When the spin is computed using only the gas, the trend with $d_{\text {skel }}$ is clearly visible, and it is absent when only the stellar component is considered.

When the distributions shown in Figure 7 were re-computed with spin-related quantities derived using only the gas component (shown in Figure B.2), indications of the possibility of using spin-related quantities to trace the cosmic web start to emerge. In particular, in the case of low $d_{\text {skel }}$ values, a deviation of $\langle\cos (4 \theta)\rangle$ from the average relative to the full box is visible. This deviation is comparable in magnitude to the deviation of the (specific)SFR. In the case of other spin-related quantities or other distances $\left(d_{\mathrm{CP}}\right.$ and $\left.d_{\mathrm{fil}}\right)$, no significant variation is visible. 


\section{Gas component}

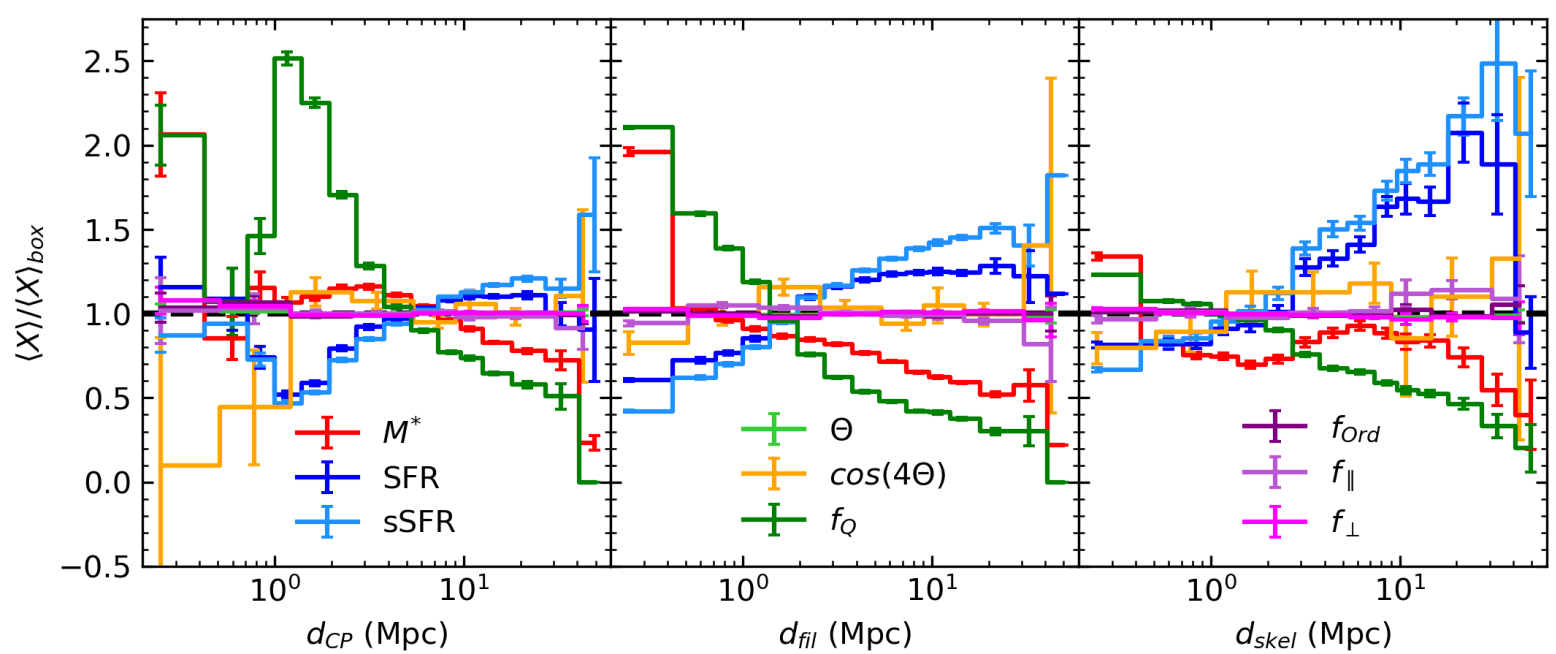

Fig. B.2. Normalised distributions (expressed as $\frac{\langle X\rangle}{\langle X\rangle_{b o x}}$ ) with respect to distances $d_{\mathrm{CP}}$ (left panel), $d_{\text {fil }}$ (middle panel), and $d_{\text {skel }}$ (right panel). The quantities considered in each panel $(\langle X\rangle)$ are respectively: $\left\langle M^{*}\right\rangle$ (red), $\langle$ SFR $\rangle$ (dark blue), $\langle$ sSFR $\rangle$ (light blue), $\langle\theta\rangle$ (light green), $\langle\cos (4 \theta)\rangle($ orange), $f_{\mathrm{Q}}$ (dark green), $f_{\text {Ord }}$ (dark purple), $f_{\|}$(light purple), and $f_{\perp}$ (magenta). $\langle X\rangle$ indicates the average of the quantity taken including all subhaloes in the box (for $\left.d_{\text {fil }}\right)$, only those outside filaments $\left(d_{\mathrm{CP}}\right)$, and only those inside filaments $\left(d_{\text {skel }}\right)$ over the full simulation volume. Only the gas component is used to compute spin-related quantities. In every panel, the first distance bin considered extends all the way to zero for all distances, but it is cut because the $x$-axis is in logarithmic scale.

\section{Appendix C: Further checks of the use of the Bullock parameter}

The Bullock et al. (2001) parameter, as currently defined in Section 3.2.2, makes use of global quantities, meaning that the radius $R$, specific angular momentum $j$, and circular velocity $V_{c}$ entering equation (2) are computed considering all particles that are bound to each subhalo, as provided by the IllustrisTNG subhalo catalogue. A more proper way to compute the Bullock parameter would be to use virial quantities for the subhaloes. Galárraga-Espinosa et al. (2020) computed the density profiles for the subhaloes, estimating $R_{200}$ and $M_{200}$ for all the subhaloes in the catalogue. We recomputed the specific angular momentum $j_{200}$ using only particles within the sphere of radius $R_{200}$ centred on each subhalo. With these quantities, we computed $\lambda_{200}$ following equation (2). We used the newly computed quantities $j_{200}$ and $\lambda_{200}$ to perform two checks: we re-derived the distributions of spin-related quantities using $j_{200}$ (shown in Figure 6), and we divided between high-spin parameter and low-spin parameter galaxies using $\lambda_{200}$ (i.e. the result shown in Figure 14).

Figure C. 1 shows the distributions of $\langle\theta\rangle,\langle\cos (4 \theta)\rangle$, fraction of ordered galaxies $\left(f_{\text {Ord }}\right)$, fraction of parallel galaxies $\left(f_{\|}\right)$, and fraction of perpendicular galaxies $\left(f_{\perp}\right)$ as a function of $d_{\mathrm{fil}}, d_{\mathrm{CP}}$, and $d_{\text {skel }}$ for the total galaxy population, when the spin of subhaloes was computed using only particles within the subhaloes' $R_{200}$. The distributions for spin-related quantities do not show any major difference with the case in which the spin was computed using all bounded particles in the subhaloes shown in the main text.

Figure C. 2 shows the distributions of galaxy quantities as a function of the distances to the cosmic web elements for highspin parameter galaxies (i.e. high $\lambda_{200}$ ) and separated into highand low-mass. No major differences can be seen with respect to the case in which the Bullock parameter was computed using all the particles bound to subhaloes. In this case, the spin-related quantities were computed using all the bound particles to the subhaloes, in order to be consistent with what we described in the main text. Particles within $R_{200}$ were used only to compute the Bullock parameter in order to separate low-spin parameter and high-spin parameter galaxies. Figure C.3 shows an analogous of Figure 14 only for high-spin parameter galaxies, selected using $\lambda_{200}$. Again, no difference with respect to the general case in which all particles bound to a subhalo are used to measure the Bullock parameter can be identified. 

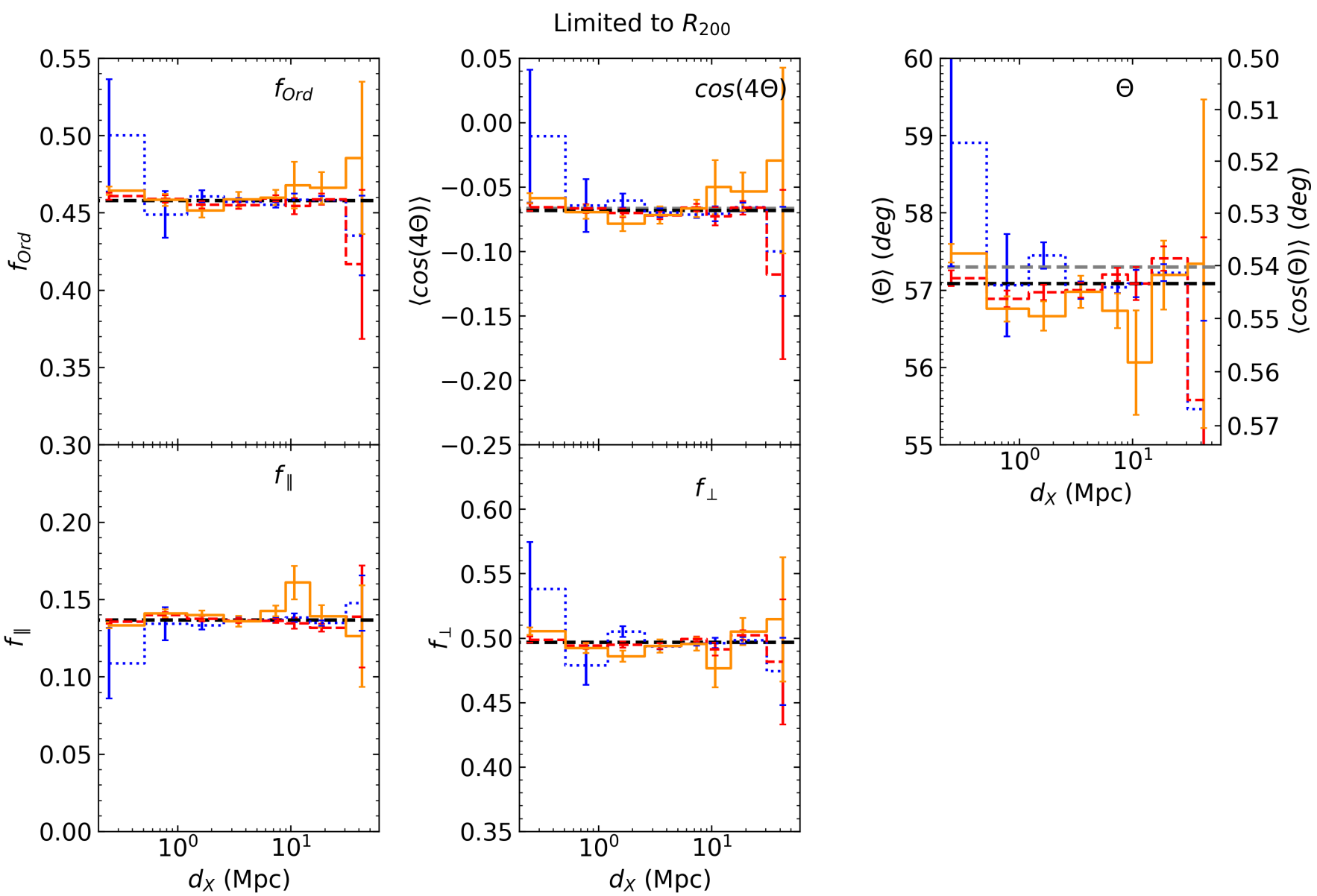

Fig. C.1. Distributions of $\langle\theta\rangle,\langle\cos (4 \theta)\rangle$, fraction of ordered galaxies $\left(f_{\text {Ord }}\right)$, fraction of parallel galaxies $\left(f_{\|}\right)$, and fraction of perpendicular galaxies $\left(f_{\perp}\right)$ as a function of the distances from the features of the cosmic web $d_{\text {fil }}$ (dashed red line in every panel), $d_{\mathrm{CP}}$ (dotted blue line in every panel), and $d_{\text {skel }}$ (solid orange line in every panel). In the case of $d_{\mathrm{CP}}$ and $d_{\text {skel }}$, only galaxies outside of filaments $\left(d_{\text {fil }} \geq 1 \mathrm{Mpc}\right)$ and inside filaments $\left(d_{\text {fil }} \leq 1 \mathrm{Mpc}\right)$ have been considered, respectively. Error bars on the distributions have been computed through bootstrap resampling. The dashed black line in every panel is the average of the considered quantity in the full simulation box. In the top left and top middle panels, the grey line is $\bar{\theta}$ and $\overline{\cos (4 \theta)}$, computed given equations (3) and (4) for the total subhalo sample. In this figure, the spin $\boldsymbol{j}_{200}$ has been computed using only particles within the $R_{200}$ of every subhalo. In every panel, the first distance bin considered extends all the way to 0 for all distances, but it is cut because the $x$-axis is in logarithmic scale. In the $f_{\|}$and $f_{\perp}$ cases, the y-axes of the plots cover very different ranges. 
High-spin parameter, limited to $R_{200}$
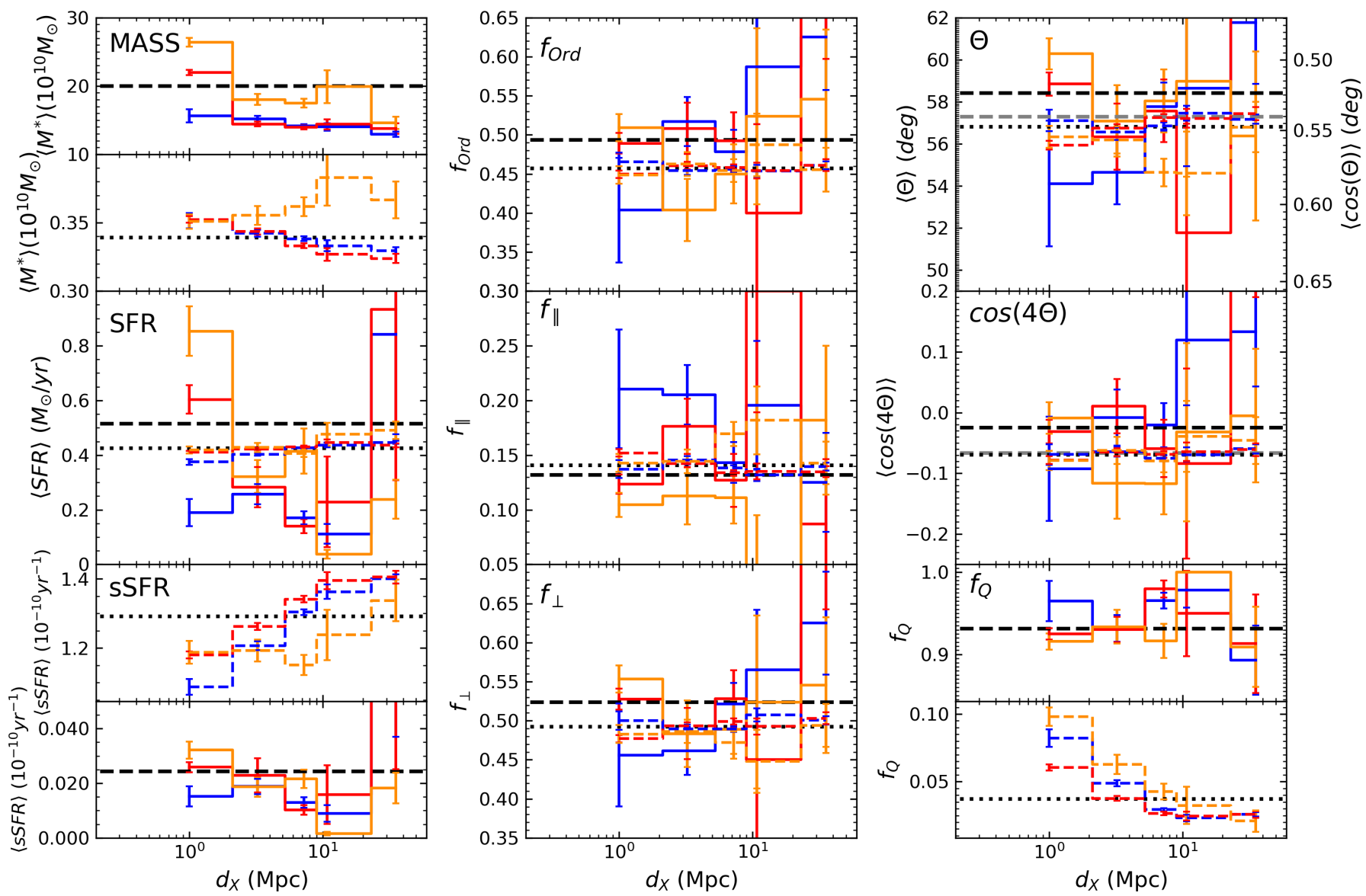

Fig. C.2. Distributions of $\left\langle M^{*}\right\rangle,\langle\mathrm{SFR}\rangle,\langle\mathrm{sSFR}\rangle,\langle\theta\rangle,\langle\cos (4 \theta)\rangle$, fraction of quenched galaxies $\left(f_{\mathrm{Q}}\right)$, fraction of ordered galaxies $\left(f_{\text {Ord }}\right)$, fraction of parallel galaxies $\left(f_{\|}\right)$, and fraction of perpendicular galaxies $\left(f_{\perp}\right)$ as a function of the distances from the features of the cosmic web $d_{\text {fil }}($ red line in every panel), $d_{\mathrm{CP}}$ (blue line in every panel), and $d_{\text {skel }}$ (orange line in every panel) and split between high- and low-mass galaxies. In the case of $d_{\mathrm{CP}}$ and $d_{\text {skel }}$, only galaxies outside of filaments $\left(d_{\mathrm{fil}} \geq 1 \mathrm{Mpc}\right)$ and inside filaments $\left(d_{\mathrm{fil}} \leq 1 \mathrm{Mpc}\right)$ have been considered, respectively. Error bars on the distributions have been computed through bootstrap resampling. The dashed (dotted) black line in every panel is the average of the considered quantity in the full simulation box considering only high-mass (low-mass) galaxies. In the top and middle panel of the central column, the grey line is $\bar{\theta}$ and $\overline{\cos (4 \theta)}$, computed given equations (3) and (4) for the total subhalo sample. In each panel, solid lines refer to high-mass galaxies and dashed lines to low-mass galaxies. In the case of mass, sSFR, and $f_{\mathrm{Q}}$, the panel has been split into two to take into account the very different ranges on the $y$-axis occupied by the distributions. Only high-spin parameter subhaloes are used to derive the distributions selected using the Bullock parameter computed using only particles within the $R_{200}$ of subhaloes. In every panel the first distance bin considered extends all the way to zero for all distances, but it is cut because the $x$-axis is in logarithmic scale. In the $f_{\|}$and $f_{\perp}$ cases, the y-axes of the plots cover very different ranges. 


\section{Low-mass, high-spin parameter, limited to $R_{200}$}

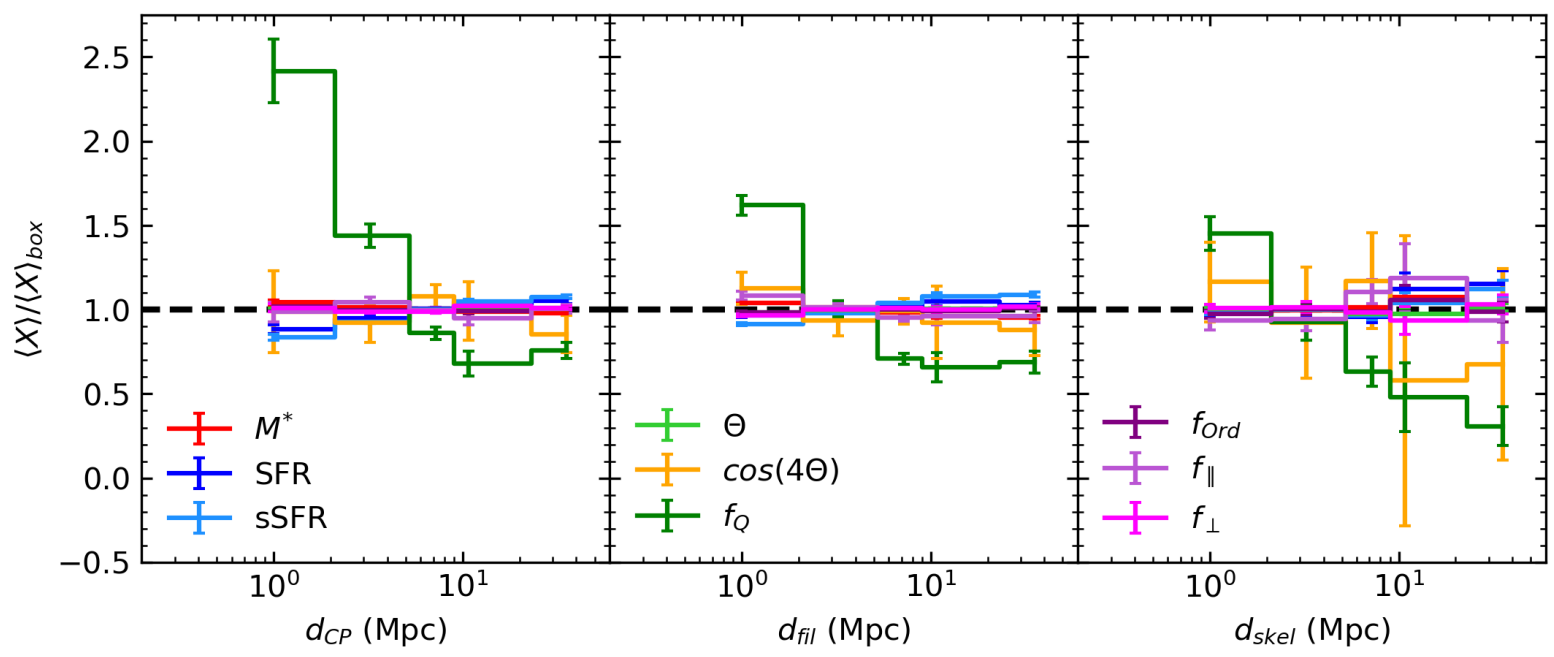

Fig. C.3. Normalised distributions (expressed as $\frac{\langle X\rangle}{\langle X\rangle_{b o x}}$ ) with respect to distances $d_{\mathrm{CP}}$ (left panel), $d_{\text {fil }}$ (middle panel), and $d_{\text {skel }}$ (right panel). The quantities considered in each panel $(\langle X\rangle)$ are respectively: $\left\langle M^{*}\right\rangle$ (red), $\langle$ SFR $\rangle$ (dark blue), $\langle$ sSFR $\rangle$ (light blue), $\langle\theta\rangle$ (light green), $\langle\cos (4 \theta)\rangle($ orange), $f_{\mathrm{Q}}$ (dark green), $f_{\text {Ord }}$ (dark purple), $f_{\|}$(light purple), and $f_{\perp}$ (magenta). $\langle X\rangle_{b o x}$ indicates the average of the quantity taken including all high-spin parameter and low-mass subhaloes in the box (for $d_{\text {fil }}$ ), only those outside filaments $\left(d_{\mathrm{CP}}\right)$, and only those inside filaments $\left(d_{\text {skel }}\right)$ over the full simulation volume. Only low-mass high-spin parameter galaxies are considered, identified by means of the Bullock parameter computed using only particles within $R_{200}$. In every panel, the first distance bin considered extends all the way to zero for all distances, but it is cut because the $x$-axis is in logarithmic scale. 